\title{
EVOLUTION AND COMPETITION OF BLOCK COPOLYMER NANOPARTICLES*
}

\author{
KARL GLASNER ${ }^{\dagger}$
}

\begin{abstract}
Nanoparticle structures formed in a mixture of diblock copolymer and solvent are investigated using a three-phase density functional model and its sharp interface approximation. A wide variety of equilibria described by localized domain patterns are quantified both numerically and analytically. Competition among multiple particles is shown to occur through mass diffusion driven by differences in chemical potential, which may or may not lead to Ostwald ripening behavior. Late stage rigid body dynamics is shown to result from interaction through dipolar fields, leading to orientational alignment and long-range attraction.
\end{abstract}

Key words. copolymer, dynamics, interaction

AMS subject classifications. 74N20, 35R35, 34D15, 70E55

DOI. $10.1137 / 18 \mathrm{M} 1192809$

Introduction. Block copolymer materials can create a wide variety of microstructures resulting from a compromise between phase segregation and polymer architecture which prevents complete separation $[1,2,3,4,5]$. In the presence of a solvent phase or confinement mechanism, these materials may form structured nanoparticles. Many current and anticipated applications for block copolymer nanostructures have emerged, including synthetic nanoreactors and drug delivery systems $[4,5]$.

The self-assembly of AB-diblock copolymers within a third immiscible phase (often called "soft confinement") has been well documented in physical experiments $[6,7,8,9]$. Symmetric copolymers are often observed to form concentric or layered domain structures $[9,10,11,12]$, as well as more exotic forms $[13,14]$. An even wider array of geometries can be realized in asymmetric mixtures [15, 16, 17].

The technological challenges to nanoparticle fabrication include controlling size and morphology. Many possible mechanisms exist for influencing nanoparticle formation [18], including annealing [19], Ostwald ripening behavior [20, 21], and controlling size with mixing rates [22]. There are many open questions concerning the kinetic aspects of synthesis and the preference between competing morphologies, which motivates the current work.

Theoretical models have been successful at the replication and prediction of copolymer nanoparticle assembly $[9,23,24,25,26,27,28,29]$. The primary focus of past work has been to reproduce equilibrium morphologies seen in experiments. Outside of numerical simulation, very little work has been done to characterize quantitative aspects of equilibria or dynamic phenomena surrounding them.

One popular modeling framework for inhomogeneous polymer systems arises from density functional theory [30,31,32]. This produces systems which are derivatives of the classical Cahn-Hilliard equation [33] and include nonlocal effects of polymer stretching. As common with phase-field approaches, there is a natural limiting case where sharply defined domains of different composition form. This allows passage

\footnotetext{
*Received by the editors June 7, 2018; accepted for publication (in revised form) November 12, 2018; published electronically January 15, 2019.

http://www.siam.org/journals/siap/79-1/M119280.html

Funding: This work was supported by NSF award DMS-1514689.

${ }^{\dagger}$ Department of Mathematics, University of Arizona, Tucson, AZ 85721 (kglasner@math. arizona.edu).
} 
to a free boundary problem which describes the evolution of domain boundaries, rather than the composition itself $[27,34,35]$. This type of free boundary problem is employed here to study domain interface configurations.

Localized or confined multidomain structures have been studied previously in the context of density functional theory and its free boundary limit. This includes lamellar configurations and their stability [36, 37, 38], concentric geometries [27, 39], double bubbles [40], and dynamic phenomena [41, 42]. Numerical work using CahnHilliard-type systems has also revealed a wide range of equilibrium morphologies $[9,26]$. Stationary assemblies of multidomain structures have also been investigated in a related three-phase system [43].

This paper addresses the question of interaction among nanoparticles. A single particle in isolation may be regarded as an equilibrium configuration of the free boundary problem, subject to a constraint on its volume. The corresponding Lagrange multiplier may be identified as the chemical potential associated with total polymer composition. In the classical scenario leading to Ostwald ripening, the monotonic behavior of the chemical potential as a function of size leads to coarsening behavior. In contrast, this may or may not occur in our system. In addition, the chemical potential associated with monomer composition also drives interaction behavior among particles. This results in both positional and orientational dynamics, which may be computed explicitly.

The organization of this paper is as follows. Density functional models and their approximating free boundary problem are reviewed in section 1. Section 2 describes the equilibrium free boundary problem and derives some qualitative and quantitative properties of solutions. A reduced dynamical description of multiparticle interaction and some of its properties are derived in section 3. Late stage interactions between particles which lead to rigid body motion are studied in section 4. Finally, numerical simulations are used to confirm and illustrate theoretical findings in section 5 .

1. Density functional model and sharp interface approximation. The modeling framework employed here stems from density functional theory [30, 31, 35] for heterogeneous polymer systems. The free energy is specified as a functional of composition variables $\phi_{A}, \phi_{B}$, and $\phi_{S}$, corresponding to copolymer constituents $A$ and $B$, and a third phase $S$, representing a homopolymer or poor solvent. One of these variables can be eliminated by invoking the standard assumption of incompressibility $\phi_{A}+\phi_{B}+\phi_{S}=1$. In addition, the fraction $f \in(0,1)$ of $A$-monomer relative to the total polymer volume is prescribed. Ohta and Nonomura [44] reformulated this description using the order parameter $\boldsymbol{u}=(\Phi, \Psi)$, where

$$
\Phi=(1-f) \phi_{A}-f \phi_{B}, \quad \Psi=f \phi_{A}+(1-f) \phi_{B} .
$$

This has the advantage of decoupling the long-range interaction between the new variables.

The free energy for a system with domain $\Omega_{0} \subset \mathbb{R}^{3}$ is $[38,44]$

$$
F=\int_{\Omega_{0}} \frac{1}{\epsilon} W(\boldsymbol{u})+\frac{\epsilon}{2}\left|\nabla\left(\boldsymbol{G}^{1 / 2} \boldsymbol{u}\right)\right|^{2} d \boldsymbol{x}+\frac{\alpha}{2} \int_{\Omega_{0}} \int_{\Omega_{0}} K\left(x, x^{\prime}\right) \Phi(\boldsymbol{x}) \Phi\left(\boldsymbol{x}^{\prime}\right) d \boldsymbol{x} d \boldsymbol{x}^{\prime} .
$$

The potential $W(\boldsymbol{u})$ has minima

$$
\boldsymbol{u}_{S}=(0,0), \quad \boldsymbol{u}_{A}=(1-f, f), \quad \boldsymbol{u}_{B}=(-f, 1-f),
$$

corresponding to each pure phase. The symmetric, positive definite gradient energy tensor $\boldsymbol{G}$ and potential $W$ can be calibrated in order to prescribe interfacial energies, 
which is explained below. Lastly, the nonlocal energy term has interaction kernel $K()$, which is taken to be the Laplacian Green's function.

Generalized chemical potentials $\nu, \mu$ arise as variations of the free energy

$$
\nu=\frac{\delta F}{\delta \Phi}, \quad \mu=\frac{\delta F}{\delta \Psi} .
$$

Diffusive dynamics are driven by gradients of chemical potential resulting in

$$
\left(\begin{array}{c}
\Phi_{t} \\
\Psi_{t}
\end{array}\right)=\nabla \cdot\left[M \nabla\left(\begin{array}{c}
\delta F / \delta \Phi \\
\delta F / \delta \Psi
\end{array}\right)\right] .
$$

The mobility tensor $\boldsymbol{M}$ describes the rates of diffusion and cross-diffusion and for simplicity of presentation will be taken to be the identity.

The free boundary approximation of (5) is used as a more tractable model for our analysis. This results in the usual way (see, e.g., $[27,34,45,46])$ from the limit $\epsilon \rightarrow 0$, wherein $(\Phi, \Psi) \rightarrow\left(\Phi_{0}, \Psi_{0}\right)=\boldsymbol{u}_{A, B, S}$ almost everywhere. The limiting configuration may therefore be associated with corresponding domains $\Omega_{A, B, S}$. Polymer phase domains $\Omega_{A}$ and $\Omega_{B}$ are assumed open and bounded in what follows. The solvent phase $\Omega_{S}$ is the interior of the complement of $\Omega_{A} \cup \Omega_{B}$, which may be unbounded (depending on $\Omega_{0}$ ). The limiting free boundary problem involves the phase interfaces, denoted by $\partial \Omega_{\mathcal{I}}$, where $\mathcal{I} \in\{A B, B S, S A\}$. The normal interface velocities $V_{n}$ are prescribed by the system

$$
\begin{aligned}
\Delta \nu & =\alpha \Phi_{0}, \quad \boldsymbol{x} \in \Omega_{S} \cup \Omega_{A} \cup \Omega_{B}, \\
\Delta \mu & =0, \quad \boldsymbol{x} \in \Omega_{S} \cup \Omega_{A} \cup \Omega_{B}, \\
\nu\left[\Phi_{0}\right]+\mu\left[\Psi_{0}\right] & =-\sigma_{\mathcal{I}} \kappa, \quad \boldsymbol{x} \in \partial \Omega_{\mathcal{I}}, \\
{[\nu] } & =0=[\mu], \quad \boldsymbol{x} \in \partial \Omega_{\mathcal{I}}, \\
V_{n}\left[\Phi_{0}\right] & =-[\partial \nu / \partial n], \quad \boldsymbol{x} \in \partial \Omega_{\mathcal{I}}, \\
V_{n}\left[\Psi_{0}\right] & =-[\partial \mu / \partial n], \quad \boldsymbol{x} \in \partial \Omega_{\mathcal{I}} .
\end{aligned}
$$

The notation [] refers to the difference in a quantity on either side of the interface, i.e., the jump from an arbitrarily prescribed - phase to the + phase. By convention, the normal to the interface is oriented in the + direction, so that the interface mean curvature $\kappa$ is positive if the phase corresponding to - is locally convex. The parameters $\sigma_{\mathcal{I}}$ and $\alpha$ are surface energy and polymer energy coefficients, respectively; their specification is described in detail below.

In addition to (6)-(11), there is a Herring-Young-Laplace condition imposed at triple junctions [46], which may be stated as

$$
\sum_{\mathcal{I} \in\{A B, B S, S A\}} \sigma_{\mathcal{I}} \boldsymbol{n}_{\mathcal{I}}=0
$$

where $\boldsymbol{n}_{p q}$ denotes the normal directed from phase $p$ to phase $q$. It is useful to state this condition in terms of vectors $\boldsymbol{t}_{\mathcal{I}}=\boldsymbol{l} \times \boldsymbol{n}_{\mathcal{I}}$, where $\boldsymbol{l}$ is the tangent to the three-phase line. After taking cross products with $\boldsymbol{l},(12)$ becomes

$$
\sum_{\mathcal{I} \in\{A B, B S, S A\}} \sigma_{\mathcal{I}} \boldsymbol{t}_{\mathcal{I}}=0
$$

The primary interest here is in one or more isolated contiguous structures within a large but finite system domain $\Omega_{0}$. The problem of studying just a single structure 
in isolation can be idealized by choosing the domain to be unbounded (section 2). The interaction of many such structures can then be placed into a matched asymptotics framework (section 3.1), wherein the inner solutions are, to leading order, the infinite domain equilibria. Solvability of (6) with either periodic boundary conditions or suitable decay of $\mu$ at infinity demands that the volume fractions of $A$ and $B$ domains satisfy

$$
\frac{\left|\Omega_{A}\right|}{\left|\Omega_{B}\right|}=\frac{f}{1-f} .
$$

Numerical simulation of the free boundary problem will utilize the phase field approximation (5). The surface energy parameters $\sigma_{\mathcal{I}}$ can be associated with the potential $W$ and gradient energy tensor $\boldsymbol{G}$ by

$$
\sigma_{\mathcal{I}}=\inf _{\boldsymbol{\gamma}}\left\{\int_{0}^{1} \sqrt{W(\gamma(s))}\left|\boldsymbol{G}^{1 / 2} \boldsymbol{\gamma}^{\prime}(s)\right| d s, \quad \gamma(0)=\boldsymbol{u}_{p}, \quad \gamma(1)=\boldsymbol{u}_{q}\right\},
$$

where $\mathcal{I}=p q$ and $\boldsymbol{u}_{p}, \boldsymbol{u}_{q}$ are the potential minima for phases $p$ and $q$. This represents an extension of Baldo's minimal path formula [47], wherein the equilibrium interface profiles $\gamma(s)$ are characterized as geodesics with respect to a degenerate metric. A convenient choice for $\boldsymbol{G}$ and $W$ which satisfies this characterization was proposed by Boyer and Lapuerta [48]. For a reference system with potential minima $\tilde{\boldsymbol{u}}=(0,0)$, $(1,0)$, and $(0,1)$, they set

$$
\tilde{\boldsymbol{G}}=\left(\begin{array}{cc}
\Sigma_{1}+\Sigma_{3} & \Sigma_{3} \\
\Sigma_{3} & \Sigma_{2}+\Sigma_{3}
\end{array}\right)
$$

and

$\tilde{W}(\tilde{\boldsymbol{u}})=\sigma_{A B} \tilde{\boldsymbol{u}}_{1}^{2} \tilde{\boldsymbol{u}}_{2}^{2}+\sigma_{A S} \tilde{\boldsymbol{u}}_{1}^{2}\left(1-\tilde{\boldsymbol{u}}_{1}-\tilde{\boldsymbol{u}}_{2}\right)^{2}+\sigma_{B S} \tilde{\boldsymbol{u}}_{2}^{2}\left(1-\tilde{\boldsymbol{u}}_{1}-\tilde{\boldsymbol{u}}_{2}\right)^{2}+\Lambda \tilde{\boldsymbol{u}}_{1}^{2} \tilde{\boldsymbol{u}}_{2}^{2}\left(1-\tilde{\boldsymbol{u}}_{1}-\tilde{\boldsymbol{u}}_{2}\right)^{2}$,

where $\Sigma_{1}=\sigma_{A B}+\sigma_{A S}-\sigma_{B S}, \Sigma_{2}=\sigma_{A B}+\sigma_{B S}-\sigma_{A S}, \Sigma_{3}=\sigma_{A S}+\sigma_{B S}-\sigma_{A B}$, and $\Lambda$ is a suitably large adjustable parameter. To obtain a potential which has minima as in (3), the corresponding gradient tensor and potential are obtained by a linear transformation

$$
\boldsymbol{G}=\boldsymbol{Q}^{T} \tilde{\boldsymbol{G}} \boldsymbol{Q}, \quad W(\boldsymbol{u})=\tilde{W}(\boldsymbol{Q u}), \quad \boldsymbol{Q}=\frac{1}{f^{2}+(1-f)^{2}}\left(\begin{array}{cc}
1-f & f \\
-f & 1-f
\end{array}\right) .
$$

The parameters $\epsilon$ and $\alpha$ in (2) correspond to physical lengthscales. As in other diffuse interface problems, $\epsilon$ is proportional to the interface width and is set to unity for convenience. A scaling argument [49] suggests that equilibrium domain structures will have a characteristic width (defined in the narrow direction) proportional to $\alpha^{-1 / 3}$. To achieve a separation of lengthscales required in the small $\epsilon$ limit, one needs $\alpha \gg \epsilon^{-3}$. For later numerical computation, $\alpha$ is fixed at $2 \times 10^{3}$, ensuring that the sharp interface limit is respected.

The system (5) describes two types of phase segregation. On one hand, microphase segregation between monomer components A and B leads to spatial inhomogeneity of the order parameter $\Phi$. This is ultimately inhibited by polymer stretching, encoded by the nonlocal energy term. Similarly, phase segregation between polymer phases and solvent occurs, leading to inhomogeneity of $\Psi$. When the polymer occupies only a small fraction of the domain, the combined effect of phase segregation 
processes is to create nanoparticles composed of patterned A and B phase domains, whose morphology is highly dependent on surface energies, composition, and size. In the absence of polymer effects, the phase segregation process would lead to Ostwald ripening, wherein small particles shrink at the expenses of larger ones. It will be demonstrated that this process is altered both quantitatively and qualitatively in the presence of microphase segregation.

2. Localized equilibria. The fundamental objects in this study are nanoparticle equilibria, which are stationary solutions to the free boundary problem. A wide variety of such configurations have been observed $[9,27]$. These structures are in equilibrium with their local environment, in the sense that in isolation the chemical potential $\mu$ which drives polymer mass diffusion is constant everywhere. This section describes and quantifies some basic types of domain configurations.

2.1. Mathematical formulation and morphological classes. The equilibrium configurations of interest may be characterized as a pair of bounded, open domains $\Omega=\left(\Omega_{A}, \Omega_{B}\right)$ with piecewise smooth boundaries, satisfying (14), and comprising a simply connected set. These satisfy the stationary version of the problem (6)-(13), which reads as

$$
\begin{aligned}
\Delta \nu & =\alpha \Phi_{0}, \quad \boldsymbol{x} \in \Omega_{S} \cup \Omega_{A} \cup \Omega_{B}, \\
\nu\left[\Phi_{0}\right]+\mu_{\infty}\left[\Psi_{0}\right] & =-\sigma_{\mathcal{I}} \kappa, \quad \boldsymbol{x} \in \partial \Omega_{I}, \\
{[\nu] } & =0=[\partial \nu / \partial n], \quad \boldsymbol{x} \in \partial \Omega_{I}, \\
\sum_{\mathcal{I} \in\{A B, A S, B S\}} \sigma_{\mathcal{I}} \boldsymbol{n}_{\mathcal{I}} & =0, \quad \boldsymbol{x} \in \partial \partial \Omega,
\end{aligned}
$$

with $\partial \partial \Omega$ denoting three-phase interfaces. As an idealization of a situation where an equilibrium domain configuration is far from other structures, this problem is studied on the infinite domain $\mathbb{R}^{3}$. This is exactly the leading order problem which arises when considering interaction of well-separated particles section 3.1.

Note that the field $\mu \equiv \mu_{\infty}$ is a constant here. It is shown below that this can be regarded as a Lagrange multiplier associated with a constraint on polymer domain size. The latter is a conveniently characterized by the functional

$$
M=\Sigma(\Omega) \equiv \int_{\Omega_{0}} \Psi_{0} d \boldsymbol{x}=f\left|\Omega_{A}\right|+(1-f)\left|\Omega_{B}\right|=\left[f^{2}+(1-f)^{2}\right]\left|\Omega_{A} \cup \Omega_{B}\right|,
$$

where (14) was used. In other words, $M$ is proportional to the total volume occupied by the polymer phases.

In general, explicit solutions to (19)-(22) are not easily obtained. Much more can be said about certain distinct classes, such as concentric domains patterns [27]. We define a morphological class $Q$ to be a differentiable (in the sense defined below) family $\Omega_{Q}(M)$ of topologically equivalent equilibria with $\Sigma\left(\Omega_{Q}(M)\right)=M$. For a particular morphological class $Q$, there is assumed to be a smooth dependence of equilibrium chemical potential $\mu_{\infty}$ on size $M$,

$$
\mu_{\infty}=\mu_{\infty}(M ; Q)
$$

For any particular configuration $\Omega$ (not necessarily at equilibrium), an admissible perturbation $\tilde{\Omega}$ corresponds to a normal displacement of the domain boundaries which maintains the volume fraction constraint, as well as the coherence of three-phase 
junctions. Denoting the collection of such boundaries as $\partial \Omega$, this is a smooth mapping $\tilde{\Omega}: \partial \Omega \rightarrow \mathbb{R}$ constrained to respect (14), so that

$$
\int_{\partial \Omega}\left[\Phi_{0}\right] \tilde{\Omega} d \boldsymbol{x}=0
$$

In addition, the interfaces must move so that they all intersect at three-phase junctions. Denoting the infinitesimal motion of the junction in a plane normal to the three-phase line by $\boldsymbol{q}$, it follows that

$$
\tilde{\Omega}=\boldsymbol{n}_{\mathcal{I}} \cdot \boldsymbol{q}, \quad \mathcal{I} \in\{A B, A S, B S\} .
$$

This represents an overdetermined linear system for $\boldsymbol{q}$, whose solvability constrains the values of $\tilde{\Omega}$ at the three-phase junction.

Lastly, a family of equilibrium configurations $\Omega(\beta)$ is said to be differentiable on some open interval $\beta_{1}<\beta<\beta_{2}$ if each interface in $\partial \Omega(\beta)$ can be locally described by a smooth parameterization $\boldsymbol{x}=\boldsymbol{x}(s ; \beta)$. In this case, we may define $d \Omega / d \beta$ to be an admissible perturbation, where

$$
\frac{d \Omega}{d \beta}(\boldsymbol{x}(s ; \beta)) \equiv \frac{d \boldsymbol{x}}{d \beta}(s ; \beta) .
$$

2.2. Variational characterization of equilibria. As in other phase field systems, the sharp interface problem (19)-(22) has a natural energy, arising from (2) as the $\Gamma$-limit (for the two phase problem, see, e.g., [50]). This energy may be decomposed as $E=E_{s}+E_{p}$, where $E_{s}$ is the sum of surface energies,

$$
E_{s}=\sum_{\mathcal{I} \in\{A B, A S, B S\}} \sigma_{\mathcal{I}}\left|\partial \Omega_{\mathcal{I}}\right|,
$$

and $E_{p}$ is the energy of polymer stretching,

$$
E_{p}=\frac{\alpha}{2} \int_{\mathbb{R}^{3}} \int_{\mathbb{R}^{3}} K\left(x, x^{\prime}\right) \Phi_{0}(\boldsymbol{x}) \Phi_{0}\left(\boldsymbol{x}^{\prime}\right) d \boldsymbol{x} d \boldsymbol{x}^{\prime} .
$$

Configurations satisfying (19)-(22) may be viewed as critical points of the augmented energy $\tilde{E}=E_{s}+E_{p}-\mu_{\infty} \Sigma$. This follows by computing the variation of $\tilde{E}$ with respect to an admissible perturbation $\tilde{\Omega}$ :

$$
\langle\delta \tilde{E}, \tilde{\Omega}\rangle \equiv \int_{\partial \Omega}\left(\nu\left[\Phi_{0}\right]+\sigma_{\mathcal{I}} \kappa+\mu\left[\Psi_{0}\right]\right) \tilde{\Omega} d \boldsymbol{x}+\sum_{\mathcal{I} \in\{A B, B S, S A\}} \int_{\partial \partial \Omega} \sigma_{\mathcal{I}}\left(\boldsymbol{q} \cdot \boldsymbol{t}_{\mathcal{I}}\right) d \boldsymbol{x} .
$$

The triple junction condition (22) arises as a natural boundary condition (in the calculus of variations sense). The first term in (30) is zero for all admissible $\tilde{\Omega}$; the latter set is simply the orthogonal complement of $\left[\Phi_{0}\right]$ by $(25)$. It follows that for some constant $c$,

$$
\nu\left[\Phi_{0}\right]+\sigma \kappa+\mu\left[\Psi_{0}\right]=c\left[\Phi_{0}\right] .
$$

Redefining $\nu$ as $\nu-c$ leads to the equilibrium system (19)-(22).

The variational formulation can now be used to characterize the relationship between particle size and chemical potential in two different ways. First, suppose 
that $\Omega_{Q}(M)$ is a given morphological class of equilibria. Differentiation of $E\left(\Omega_{Q}(M)\right)$ with respect to $M$ gives

$$
\frac{d E}{d M}=\left\langle\delta \tilde{E}, d \Omega_{Q} / d M\right\rangle+\mu_{\infty}(M)\left\langle\delta \Sigma, d \Omega_{Q} / d M\right\rangle=\mu_{\infty}(M) .
$$

This is just a restatement of the thermodynamic definition of chemical potential as the derivative of free energy with respect to volume.

A second characterization of $\mu_{\infty}(M)$ can be made as follows. Given some equilibrium configuration $\Omega(1)$, consider the differentiable family $\Omega(r)$ of dilations, i.e., configurations $\Omega_{A}(r)$ and $\Omega_{B}(r)$ defined so that $\boldsymbol{x} \in \Omega_{A, B}(1)$ if and only if $r \boldsymbol{x} \in \Omega_{A, B}(r)$. By virtue of the scaling of individual energy components (in three dimensions),

$$
\tilde{E}(\Omega(r))=r^{2} E_{s}(\Omega(1))+r^{5} E_{p}(\Omega(1))-\mu_{\infty} r^{3} .
$$

Differentiating with respect to $r$ and setting $r=1$ gives

$$
\left.\langle\delta \tilde{E}(\Omega(r)), d \Omega(r) / d r\rangle\right|_{r=1}=2 E_{s}(\Omega(r))+5 E_{p}(\Omega(1))-3 \mu_{\infty} \Sigma(\Omega(1))
$$

or, in other words,

$$
\mu_{\infty}=\frac{2 E_{s}+5 E_{p}}{3 M}
$$

In general, the quantitative relation among energy components, size, and geometry is complicated. For large particles, on the other hand, subdomains appear to have a characteristic width independent of their size. This suggests an "equipartition hypothesis": for large, low energy domain equilibria, the surface and polymer energy contributions are each proportional to particle size. Therefore, the formula (35) says that $\mu_{\infty}$ should approach, or at least be bounded from below by, a constant as the size becomes large. Computations in section 5.3 are consistent with this conclusion.

2.3. Micelle equilibria. One tractable class of structures which admits analytic solutions are two-domain "micelles," where the domain boundaries $\partial \Omega_{A B}$ and $\partial \Omega_{B S}$ are concentric spherical shells with radii $r_{1}$ and $r_{2}$. This means $r_{1}=f^{1 / 3} r_{2}$ by virtue of (14).

The solution of (19) with condition (20) for $r<r_{1}$ gives

$$
\nu=\frac{\alpha(1-f)\left(r^{2}-r_{1}^{2}\right)}{2 d}+\frac{2 \sigma_{A B}}{r_{1}}+(1-2 f) \mu_{\infty},
$$

whereas the solution for $r_{1}<r<r_{2}$ is

$$
\nu=\frac{-\alpha f\left(r^{2}-r_{1}^{2}\right)}{2 d}+c\left(1 / r_{1}-1 / r\right)+\frac{2 \sigma_{A B}}{r_{1}}+(1-2 f) \mu_{\infty} .
$$

The constant $c$ is determined by applying the second equality of (21) on the $\partial \Omega_{A B}$ interface, giving $c=\alpha r_{1}^{3} / 3$. Finally, applying (20) gives

$$
\frac{-\alpha f\left(r_{2}^{2}-r_{1}^{2}\right)}{2 d}+\alpha r_{1}^{d}\left(1 / r_{1}-1 / r_{2}\right)+\frac{2 \sigma_{A B}}{r_{1}}+(1-2 f) \mu_{\infty}=\frac{(1-f) \mu_{\infty}}{f}-\frac{2 \sigma_{B S}}{f r_{2}},
$$

which implies

$$
\mu_{\infty}=\frac{f}{f^{2}+(1-f)^{2}}\left(\frac{2\left(\sigma_{A B} f^{-1 / 3}+\sigma_{B S} f^{-1}\right)}{r_{2}}+\frac{\alpha r_{2}^{2}\left(2 f^{2 / 3}+f^{5 / 3}-3 f\right)}{6}\right),
$$


where

$$
r_{2}=\left(\frac{4 M}{3 \pi\left(f^{2}+(1-f)^{2}\right)}\right)^{1 / 3} .
$$

It is important to observe that the relationship (39) between chemical potential and size is not monotonic. In particular, $\mu_{\infty}(M)$ has a local minimum corresponding to outer radius

$$
r_{\text {min }}=\left(\frac{6\left(\sigma_{A B} f^{-1 / 3}+\sigma_{B S} f^{-1}\right)}{\alpha\left(2 f^{2 / 3}+f^{5 / 3}-3 f\right)}\right) .
$$

2.4. Multiple bubble equilibria. Another important morphological class is one where all $A$ - and $B$-subdomains are adjacent to the solvent phase. We call these bubble configurations, and they are commonly observed in simulations where the monomer volume fraction is $\approx 1 / 2$ (see, e.g., Figure 4 ).

The simplest of these structures is the two-domain bubble. In the absence of the polymer energy term (i.e., $\alpha=0$ ), equilibria solve a well-known minimal surface problem. The solution is geometrically simple: interfaces are two parts of a sphere, joined by a planar surface (this was conjectured in [51] and proved in [52]). Ren and Wei [40] showed that this solution may be used as a basis for continuation to nonzero polymer energy $\alpha>0$.

Our interest is in obtaining quantitative information about solutions to (19)-(22), and numerical continuation in the size parameter was implemented. This was done by first finding a single equilibrium along a branch of a particular morphological class by gradient descent dynamics (5) and then adiabatically increasing and decreasing the total integral of $\Psi$. The corresponding values of $\mu$ are determined by finding $\partial W / \partial \Psi$ away from diffuse interfaces.

Figure 1 shows the results of the computation for the case $\sigma_{A B}=\sigma_{B S}=\sigma_{S A}=1$ and $f=0.5$, along with the analytical results of the previous section for micelle equilibria. The apparent discontinuity in the derivative of $\mu_{\infty}(M)$ suggests that a symmetry breaking bifurcation is encountered. This corresponds to a morphological transition where the $A B$-interfaces become nonplanar as size increases. Except for small particles, there is a distinct ordering among morphological classes, where the three-bubble configurations generally have lower chemical potential than the twobubble and micelle configurations.

The situation for equal surface energies but asymmetric volume fraction $f=0.35$ was also investigated. In this case, numerical continuation was used to compute twobubble configurations, along with their respective chemical potentials $\mu_{\infty}(M)$. The results of this computation, along with the analytical micelle results, are shown in Figure 2. As two-bubble configurations become larger, the minority phase domain becomes elongated and is mostly encapsulated by the majority phase. This continues until the minority phase domain breaks into two domains (far right depiction in Figure $5)$. In contrast to the case of symmetric volume fraction, the chemical potential for the micelle branch is generally smaller than those for two bubbles.

3. Competition via mass diffusion. When there is more than one equilibrium domain configuration in a system, differences in chemical potential may drive mass diffusion, resulting in dynamic evolution of particles themselves. This idea can be made precise by extending classical LSW theory $[53,54]$ to the present model. In this framework, a separation of lengthscales is assumed between the interdomain spacing and the characteristic width of domains. In addition, it is supposed that equilibrium 


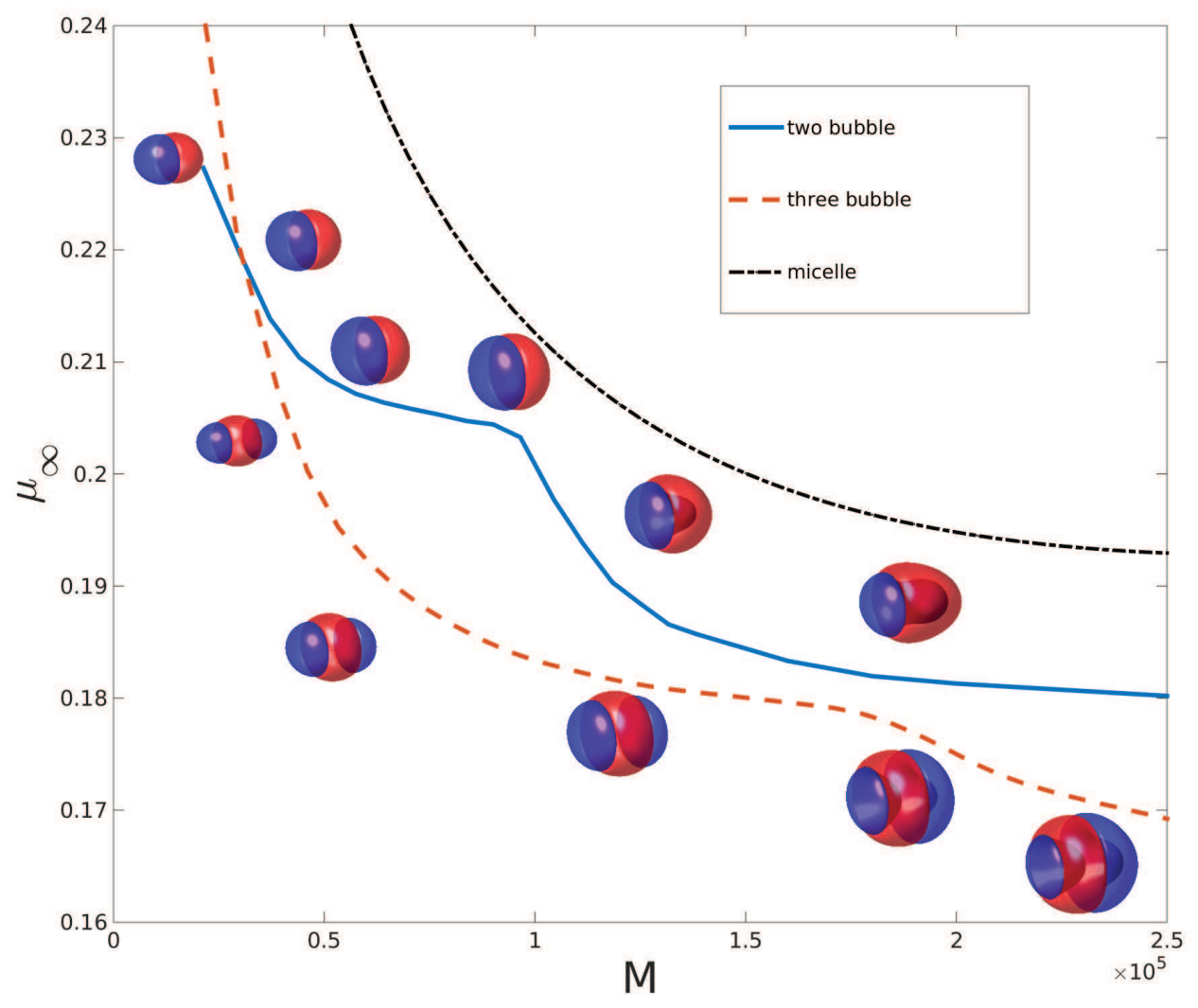

FIG. 1. The relationship between $\mu_{\infty}$ and size $M$ for bubble morphologies with two (solid/blue) and three (dashed/red) domains. Some representative equilibrium configurations are displayed along each graph. For comparison, the relationship for micelles is also shown (dash-dot/black). The surface energies were all set to one, and the volume fraction was $f=0.5$.

domain configurations evolve adiabatically: the influence of diffusion between particles is slow compared to the timescale of relaxation to a new equilibrium configuration. The relaxation rate can in principle be identified by linearization of the dynamic equations and finding the smallest (i.e., slowest) eigenvalue. This spectral problem has been carried out for certain symmetric configurations [27] but is in general complicated to characterize. It is also possible that diffusive interaction might drive morphological changes (see section 5.1), either through size evolution or coalescence. These effects are not incorporated in what follows.

3.1. The small volume fraction limit. Suppose that the system contains multiple equilibrium domains $\Omega_{i}=\left(\Omega_{A i}, \Omega_{B i}\right), i=1,2, \ldots, N$, with centers of mass $\boldsymbol{x}^{i}$ and sizes $M_{i}$ (superscripts will be used to index multiple vector or tensor quantities, and subscripts will only be used for components). Each particle domain configuration is assumed to be in a morphological class $Q_{i}$, and that small changes in size will adiabatically result in configurational changes within the class $Q_{i}$. The chemical potential associated with each equilibrium will be labeled $\mu_{\infty}\left(M_{i} ; Q_{i}\right)$.

We consider a system domain $\Omega_{0}$ which is large but finite. For simplicity, this 


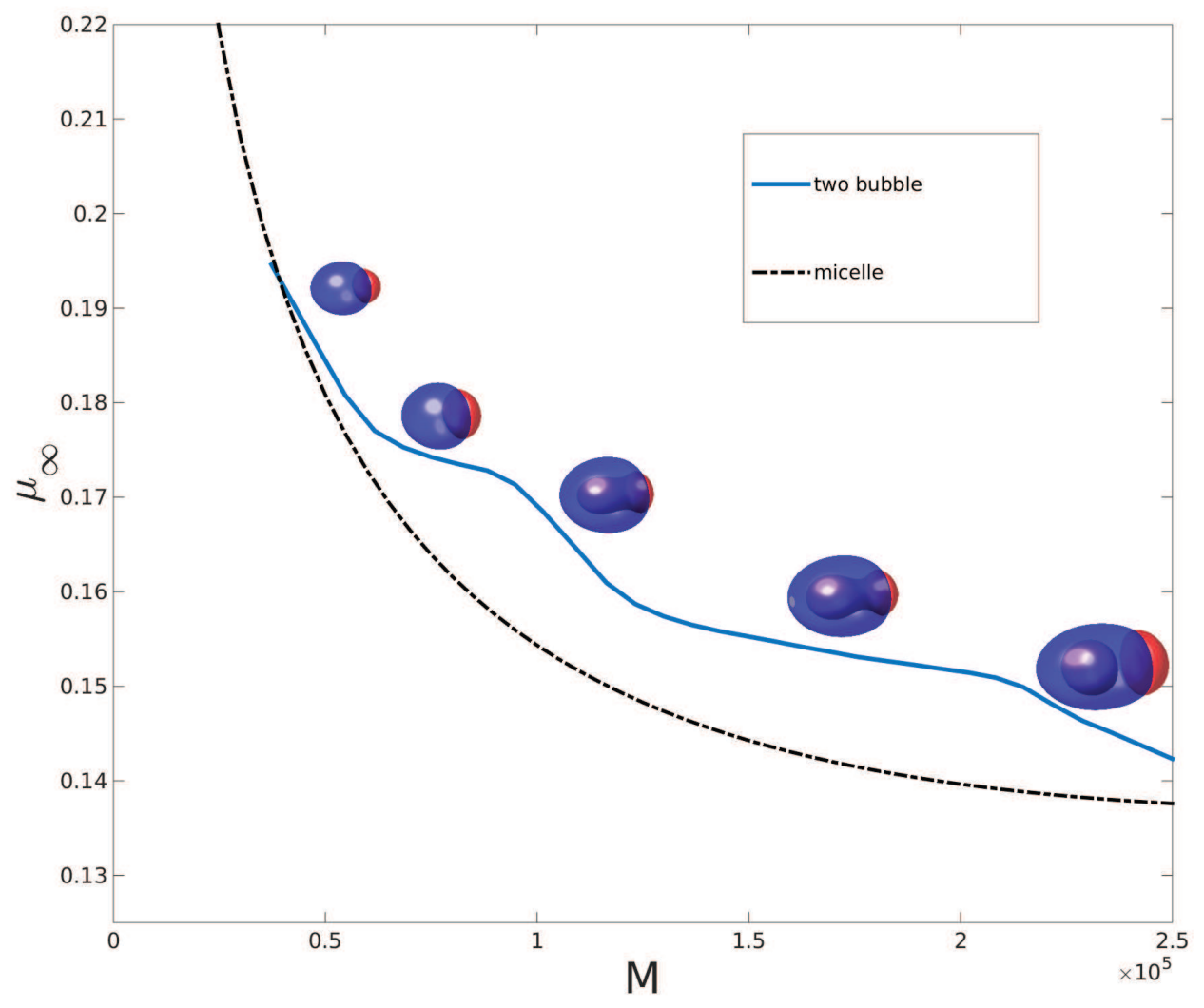

FIG. 2. The relationship between chemical potential $\mu_{\infty}$ and size $M$ for bubble morphologies with two (solid/blue) in the case of unequal volume fraction $f=0.35$, with representative equilibria displayed along the graph. For comparison, the relationship for micelles is also shown (dash-dot/black).

is taken to be a large rectangular box endowed with periodic boundary conditions. Letting $\varepsilon \ll 1$ be the ratio of domain width to interdomain separation, a scaled coordinate $\boldsymbol{y}=\varepsilon \boldsymbol{x}$ is introduced so that the scaled centers of mass $\boldsymbol{y}^{i}=\varepsilon \boldsymbol{x}^{i}$ are $O(1)$ apart. The solution away from the $\Omega_{i}$ regions is expanded: $\mu(\boldsymbol{y})=\mu_{0}(\boldsymbol{y})+\varepsilon \mu_{1}(\boldsymbol{y})+$ $\cdots$. It is found that $\mu_{0}$ is a constant, and $\mu_{1}$ solves

$$
\Delta \mu_{1}=0, \quad \boldsymbol{y} \in \Omega_{0} /\left\{\boldsymbol{y}^{1}, \boldsymbol{y}^{2}, \ldots, \boldsymbol{y}^{n}\right\}
$$

subject to periodic boundary conditions. In general, $\mu_{1}$ has singular behavior as $\boldsymbol{y} \rightarrow \boldsymbol{y}^{i}$, which will be determined.

Near the domain $\Omega_{i}$, there is a boundary layer whose solution is written in terms of the unscaled coordinate $\boldsymbol{x}$. Note that although $\boldsymbol{x}$ is finite in the original problem, the boundary layer solutions are sought in the unbounded domain $\boldsymbol{x} \in \mathbb{R}^{3}$. Since the domain boundary configurations $\partial \Omega_{i}$ are exactly of equilibrium shape, the fields $\mu$ and $\nu$ inside $\Omega_{i}$ are completely determined by the solution of (19), (20), and (22). In particular, $\mu=\mu_{\infty}\left(M_{i} ; Q_{i}\right)$ on the $S$-phase boundaries $\partial \Omega_{i}^{S}$. The leading order boundary layer problem satisfies $\Delta \mu=0$, and matching to the outer solution gives $\lim _{x \rightarrow \infty} \mu(\boldsymbol{x})=\mu_{0}$. The solution to this problem may be obtained as 
$\mu=\left(\mu_{\infty}\left(M_{i} ; Q_{i}\right)-\mu_{0}\right) U(y)+\mu_{0}$, where $U$ solves

$$
\Delta U=0, \quad U=1 \text { on } \partial \Omega_{i}^{S}, \quad U(\infty)=0 .
$$

This is a standard problem in potential theory and admits a unique solution which behaves as $U \sim C_{i} /|\boldsymbol{y}|$ for $|\boldsymbol{y}| \rightarrow \infty$. The so-called electrostatic capacity $C_{i}$ is a function of the boundary geometry and may be written as

$$
C_{i}=\frac{1}{4 \pi} \int_{\partial \Omega_{i}^{S}} \partial U / \partial n d \boldsymbol{x}
$$

where the normal to the interface is directed outward.

Returning to the outer solution, it follows that

$$
\left.\mu_{1} \sim C_{i}\left(\mu_{\infty}\left(M_{i} ; Q_{i}\right)\right)-\mu_{0}\right) /\left|\boldsymbol{y}-\boldsymbol{y}^{i}\right|, \quad \boldsymbol{y} \rightarrow \boldsymbol{y}^{i} .
$$

Defining the modified Green's function $G(\boldsymbol{y})$ as solving the problem

$$
\Delta G(\boldsymbol{y})=-\delta(\boldsymbol{y})+1 /\left|\Omega_{0}\right|,
$$

it follows that (up to an additive constant)

$$
\left.\mu_{1}=\sum_{i=1}^{N} C_{i}\left(\mu_{\infty}\left(M_{i} ; Q_{i}\right)\right)-\mu_{0}\right) G\left(\boldsymbol{y}-\boldsymbol{y}^{i}\right) .
$$

Solvability of the Laplace equation requires

$$
\left.\sum_{i=1}^{N} C_{i}\left(\mu_{\infty}\left(M_{i} ; Q_{i}\right)\right)-\mu_{0}\right)=0
$$

or

$$
\mu_{0}=\frac{\sum_{i=1}^{N} C_{i} \mu_{\infty}\left(M_{i} ; Q_{i}\right)}{\sum_{i=1}^{N} C_{i}} .
$$

The evolution of the parameter $M$ of each particle can now be determined. Using the second interface velocity condition in (11),

$$
\frac{d M_{i}}{d t}=\int_{\partial \Omega_{i}^{S}} V_{n}[\Psi] d \boldsymbol{x}=-\int_{\partial \Omega_{i}^{S}}[\partial \mu / \partial n] d \boldsymbol{x}=4 \pi C_{i}\left(\mu_{0}-\mu_{\infty}\left(M_{i} ; Q_{i}\right)\right) .
$$

In writing this, the normal to the interface and the jump [] across the interface are both directed outward.

Equations (49) and (50) give a coupled system for the evolution of $M_{i}$. The mean field $\mu_{0}$ can be interpreted as a weighted average of chemical potentials associated with each particle. Then (50) says that particles with lower chemical potential will grow at the expense of those with larger chemical potential; this is precisely the same conclusion as in classical Ostwald ripening arising from binary phase segregation. The novelty here lies in the fact that $\mu_{\infty}$ is not necessarily monotonically dependent on size, and the dependence also varies from one morphological class to another. This allows for a wider variety of phenomena which are investigated later on.

Based on the computations of the relation $\mu_{\infty}(M)$ given in sections 2.3 and 2.4, predictions of evolution among competing structures can be made. In the case of 
equal surface energies and symmetric monomer volume fractions, $\mu_{\infty}$ is monotonically decreasing within the two- and three-bubble morphological classes (see Figure 1). This means that larger particles will outcompete smaller ones. In addition, for particles of large enough size, the three-bubble morphology is favored over two-bubble and micelle configurations. Numerical experiments in section 5.1 are consistent with this prediction.

For the case of asymmetric volume fractions, the computations of section 2.4 show that micelles should generally be favored over two-bubble structures. In addition, the equilibrium chemical potential associated with the micelle morphology is not monotonic. This means that larger micelles might not necessarily grow at the expense of smaller ones, and ones with sizes near the minimum of the $\mu_{\infty}$ curve should be favored. On the other hand, it will be shown that particles are not necessarily driven to the size corresponding to the minimum itself.

3.2. Multiple particle equilibria and their stability. The dynamics specified by (49)-(50) may either drive individual particles to extinction (where $M$ goes to zero) or to a situation where many particles coexist. From (50), the latter case is characterized by the equality of chemical potentials:

$$
\mu\left(M_{i} ; Q_{i}\right)=\mu_{0}, \quad i=1,2, \ldots, N .
$$

The system (49)-(50) has an energy

$$
E=\sum_{i=1}^{N} E_{i}\left(M_{i}\right), \quad E_{i}\left(M_{i}\right) \equiv \int_{0}^{M_{i}} \mu_{\infty}\left(M^{\prime} ; Q_{i}\right) d M^{\prime} .
$$

Using (49)-(50), it is straightforward to show that $d E / d t \leq 0$. In addition, critical points of (52) subject to fixed total mass $\sum_{i=1}^{N} M_{i}$ yield (51), where $\mu_{0}$ is the Lagrange multiplier.

Stability of critical points may be investigated by studying the second variation of $E$ given by the quadratic form

$$
B\left(M_{1}^{\prime}, M_{2}^{\prime}, \ldots, M_{N}^{\prime}\right)=\sum_{i=1}^{N} \lambda_{i} M_{i}^{\prime 2}, \quad \lambda_{i} \equiv \frac{\partial \mu_{\infty}}{\partial M}\left(M_{i} ; Q_{i}\right),
$$

restricted to the subspace of mass conserving perturbations

$$
\sum_{i=1}^{N} M_{i}^{\prime}=0
$$

We say an equilibrium of (49)-(50) is stable if $B\left(M_{1}^{\prime}, M_{2}^{\prime}, \ldots, M_{N}^{\prime}\right)>0$ for all nonzero perturbations satisfying (54). Stability can be completely characterized by the derivatives $\lambda_{i}$ of chemical potentials.

Proposition 1. An equilibrium of (49)-(50) is stable if and only if either (1) $\lambda_{i}>0$ for all $i=1,2, \ldots, N$, or (2) there is exactly one $k \in\{1,2, \ldots, N\}$ with $\lambda_{k} \leq 0$ and

$$
\left|\lambda_{k}\right|<\bar{\lambda}, \quad \bar{\lambda} \equiv\left(\sum_{i \neq k} \lambda_{i}^{-1}\right)^{-1}
$$


The proof of this depends on the following result.

Lemma 1. Let $d_{j}>0$. The minimum of $\tilde{B}=\sum_{i=1}^{\tilde{N}} d_{i} v_{i}^{2}$ subject to the constraint

$$
\sum_{j=1}^{\tilde{N}} v_{i}=1
$$

is equal to

$$
\bar{d}=\left(\sum_{i=1}^{\tilde{N}} d_{i}^{-1}\right)^{-1}
$$

and is uniquely obtained, where $v_{i}=\bar{d} / d_{i}$.

Proof. The quadratic form $\tilde{B}$ is convex on the affine set given by (56), and therefore has a unique minimum, which solves $d_{i} v_{i}=L$ for some Lagrange multiplier $L$. Summing over $i$ and using (56) gives $L=\bar{d}$. Then the minimum value of $B$ is

$$
\sum_{i=1}^{\tilde{N}} d_{i}\left(\bar{d} / d_{i}\right)^{2}=\bar{d} .
$$

Proof of Proposition 1. Stability is obvious in the case of (1). If there are two distinct indices $l, m \in\{1,2, \ldots, N\}$ with $\lambda_{l} \leq 0$ and $\lambda_{m} \leq 0$, then for

$$
M_{i}^{\prime}= \begin{cases}+1, & i=l, \\ -1, & i=m, \\ 0, & i \neq l, m,\end{cases}
$$

$B\left(M_{1}^{\prime}, M_{2}^{\prime}, \ldots, M_{N}^{\prime}\right) \leq 0$. Finally, consider case (2) where only $\lambda_{k} \leq 0$. For stability, it suffices to consider perturbations with $M_{k}^{\prime}=-1$ and

$$
\sum_{i \neq k} M_{i}^{\prime}=1 \text {. }
$$

If (55) holds, the lemma shows that

$$
B\left(M_{1}^{\prime}, M_{2}^{\prime}, \ldots, M_{N}^{\prime}\right) \geq \lambda_{k}+\bar{\lambda}>0 .
$$

Conversely, if (55) does not hold, then for the perturbation

$$
M_{i}^{\prime}= \begin{cases}-1, & i=k, \\ \bar{\lambda} / \lambda_{i}, & i \neq k,\end{cases}
$$

one has

$$
B\left(M_{1}^{\prime}, M_{2}^{\prime}, \ldots, M_{N}^{\prime}\right)=\lambda_{k}+\bar{\lambda} \leq 0 .
$$

For domains within the same morphological class, the previous result indicates the possibility of a stable collection of particles, provided they have identical size $M$ and $\mu_{\infty}^{\prime}(M)>0$. The alternative is instability, where slightly larger particles grow at the expense of slightly smaller ones. Note that this process may restabilize once small particles are eliminated and enough additional mass has been accreted onto larger ones so that $\mu_{\infty}^{\prime}(M)>0$. Additionally, note that coexistence of different morphological classes is not ruled out; there may be cases where there is equality along the $\mu_{\infty}(M)$ curves, and simultaneously $\mu_{\infty}^{\prime}>0$ for each class at these values. 
3.3. Kinetic limit. In the case of large particle number, the evolution of the size distribution function $\eta=\eta(M, t)$ may be studied by a kinetic equation. For illustration purposes, we consider only the interaction among a chosen morphological class with chemical potential $\mu_{\infty}(M)$ and capacity $C(M)$. The resulting Smoluchowskitype kinetic equation corresponding to the dynamics $(50)$ is $[55,56]$

(64)

$\partial_{t} \eta+4 \pi \partial_{M}\left(\eta(M, t) C(M)\left[\mu_{0}-\mu_{\infty}(M)\right]\right)=0, \quad \mu_{0}=\frac{\int_{0}^{\infty} \eta(M, t) C(M) \mu_{\infty}(M) d M}{\int_{0}^{\infty} \eta(M, t) C(M) d M}$.

The capacity $C(M)$ is defined in (44), using the domain configuration within the morphological class having size $M$.

The total free energy for a given size distribution is

$$
E=\int_{0}^{\infty} \eta(M, t) e(M) d M, \quad e(M) \equiv \int_{0}^{M} \mu_{\infty}\left(M^{\prime}\right) d M^{\prime} .
$$

Note that (32) implies that $e(M)$ is just the energy of the equilibrium domain with size $M$. Provided that $\eta$ decays sufficiently fast as $M \rightarrow \infty$, the integral in (65) will be finite.

It is straightforward to compute

$$
\frac{d E}{d t}=\int_{0}^{\infty} e(M) \eta_{t} d M=4 \pi \int_{0}^{\infty} \eta(M, t) C(M)\left(\mu_{0}^{2}-\mu_{\infty}^{2}\right) d M .
$$

The dissipation of energy (65) can be seen by first noting that Jensen's inequality implies

$$
\mu_{0}^{2}=\left(\frac{\int_{0}^{\infty} \eta(M, t) C(M) \mu_{\infty}(M) d M}{\int_{0}^{\infty} \eta(M, t) C(M) d M}\right)^{2} \leq \frac{\int_{0}^{\infty} \eta(M, t) C(M) \mu_{\infty}^{2}(M) d M}{\int_{0}^{\infty} \eta(M, t) C(M) d M} .
$$

This can be combined with (66) to obtain $d E / d t \leq 0$. Since equality in (67) only occurs when $\eta(M, t)$ is a point distribution, it follows that the only time-independent solutions of (64) are where $\eta(M, t)=\delta\left(M-M^{*}\right)$ for any $M^{*}>0$.

To illustrate the dependence of $M^{*}$ on initial conditions, simulations of (64) are shown in Figure 3. The case of spherical micelles was used, where $M$ can be scaled so that $C(M)=R$ and $\mu_{\infty}=1 / R+R^{2}$ with $R=M^{1 / 3}$. The above proposition implies that the ensemble will be stable for $M>1 / 2$. The initial value of $\eta$ was chosen to be a Gaussian whose peak was either at $M=0.1$ or $M=0.45$. In both cases, $\eta$ evolves toward a point distribution $\eta=\delta\left(M-M^{*}\right)$, but $M^{*} \approx 0.63$ in the former case and $M^{*} \approx 0.57$ in the latter. We note that the initial condition dependence provides a mechanism for controlling the final size, which is important in practical applications [22].

4. Rigid body dynamics. In situations where multiple particles coexist, weaker interaction effects come into play at late stages. These are driven by inhomogeneities in the other chemical potential field $\nu$, which generically has a dipole character for isolated equilibria. Two consequences of this interaction are identified: (1) rotation is induced by field asymmetry, leading to orientational alignment, and (2) particles may exhibit translational dynamics so as to reduce dipole interaction energy. It is found that the latter is a weaker effect than the first, leading to a scenario where nearby particles align and later experience attraction dynamics. 

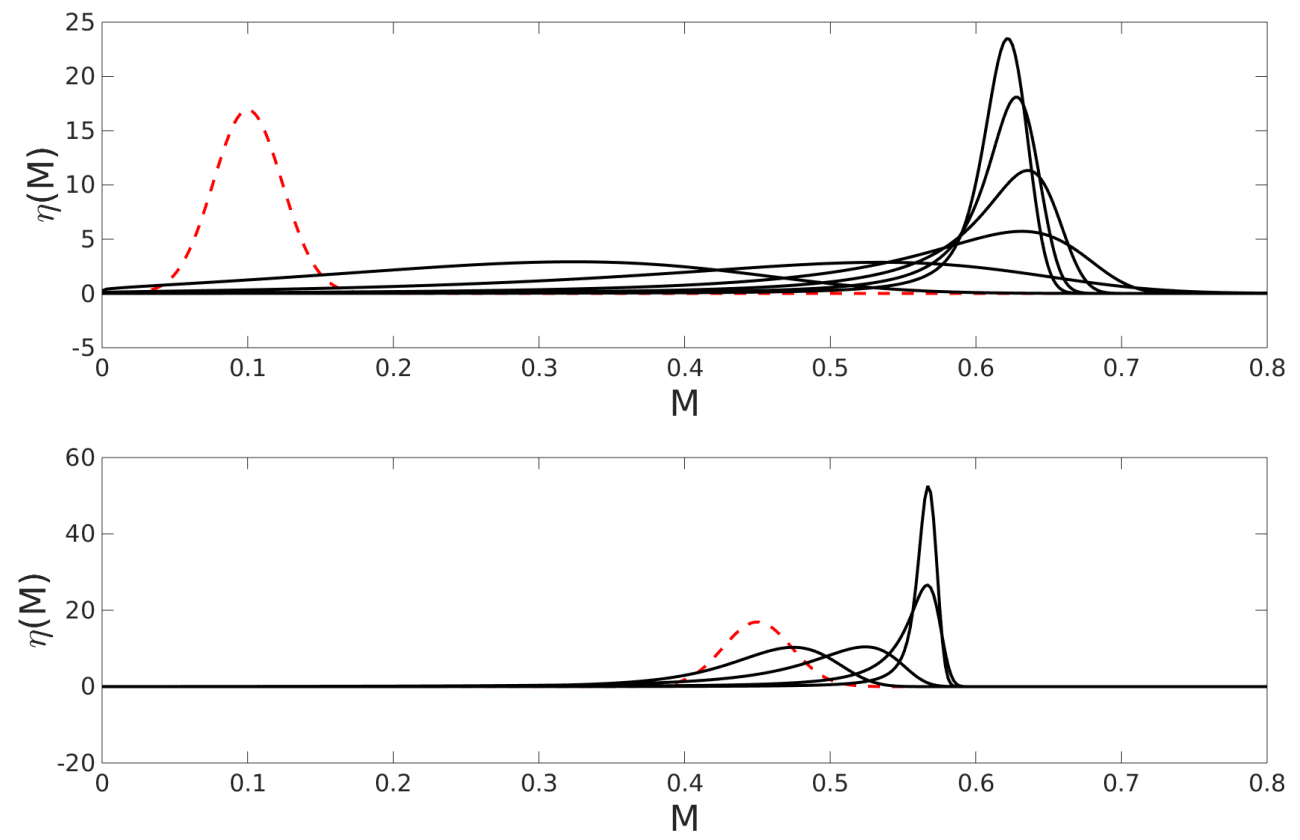

FIG. 3. Simulation of (64) for distributions with initially small radii (top) and initially large radii (bottom). Each curve has been scaled so that the integral is unity. The initial conditions are shown as red/dotted lines. In both cases, the center of mass moves right initially, and the final result is a point distribution (not shown), but at a location dependent on the initial condition.

4.1. Rotational and translational eigenfunctions. For any equilibrium configuration $\Omega$ solving (19)-(22), the field $\nu$ behaves in the far field as

$$
\nu \sim \frac{\boldsymbol{p} \cdot \boldsymbol{x}}{|\boldsymbol{x}|^{3}}, \quad \boldsymbol{x} \rightarrow \infty
$$

where $\boldsymbol{p}$ is the corresponding dipole moment. Provided that $\boldsymbol{p} \neq 0$, the associated direction $\boldsymbol{d}=\boldsymbol{p} /|\boldsymbol{p}|$ can be used as a parameter describing the orientation of the configuration.

The symmetry of the underlying model (at least for an infinite system domain) means that there is a continuous family of equilibria $\Omega(\boldsymbol{d}), \boldsymbol{d} \in S^{2}$, which are identical up to rotations about $\boldsymbol{x}=0$. For fixed $\boldsymbol{d}$, the tangent plane to the unit sphere at $\boldsymbol{d}$ is spanned by arbitrarily chosen orthonormal vectors $e^{1}, \boldsymbol{e}^{2}$. Rotation about the axis $\boldsymbol{r}_{k}=\boldsymbol{d} \times \boldsymbol{e}^{k}$ gives infinitesimal interface displacements given by

$$
\tilde{\Omega}_{k}^{R}=\left(\boldsymbol{r}_{k} \times \boldsymbol{x}\right) \cdot \boldsymbol{n}
$$

The corresponding perturbation of $\nu$ is

$$
\nu_{k}^{R}(\boldsymbol{x} ; \boldsymbol{d})=\nabla \nu \cdot\left(\boldsymbol{r}_{k} \times \boldsymbol{x}\right)
$$

It is straightforward to check that $\tilde{\Omega}_{k}^{R}$ and $\nu_{k}^{R}$ solve the the linearized equilibrium 
problem

$$
\begin{aligned}
& \Delta \nu_{k}^{R}=0, \quad \boldsymbol{x} \in \Omega_{S} \cup \Omega_{A} \cup \Omega_{B}, \\
& \nu_{k}^{R}\left[\Phi_{0}\right]=\left\{(-\partial \nu / \partial n)+\sigma_{\mathcal{I}}\left(\Delta_{s}+\kappa_{1}^{2}+\kappa_{2}^{2}\right)\right\} \tilde{\Omega}_{k}^{R}, \quad \boldsymbol{x} \in \partial \Omega_{\mathcal{I}}, \\
& {\left[\partial \nu_{k}^{R} / \partial n+\left(\partial^{2} \nu / \partial n^{2}\right) \tilde{\Omega}_{k}^{R}\right]=0, \quad \boldsymbol{x} \in \partial \Omega_{\mathcal{I}},} \\
& \quad \sum_{\mathcal{I} \in\{A B, B S, S A\}} \sigma_{\mathcal{I}} \nabla_{s} \tilde{\Omega}_{k}^{R}(\mathcal{I})=0, \quad \boldsymbol{x} \in \partial \partial \Omega .
\end{aligned}
$$

Here $\Delta_{s}$ and $\nabla_{s}$ are the surface Laplacian and gradient, and $\kappa_{1,2}$ are the principal curvatures. The notation $\tilde{\Omega}_{k}^{R}(\mathcal{I})$ refers to the limit as the triple line is approached from interface $\mathcal{I}$. Equation (73) may be simplified by noting that in a coordinate system fitted to the boundary $\partial \Omega$,

$$
\Delta \nu=\nu_{r r}+\kappa \nu_{r}+\Delta_{s} \nu=\Phi_{0},
$$

where $r$ is the normal coordinate. Using (21), the jump of this quantity across the interface is

$$
[\Delta \nu]=\left[\nu_{r r}\right]+\kappa\left[\nu_{r}\right]+\left[\Delta_{s} \nu\right]=\left[\nu_{r r}\right]
$$

so that (73) can be written as

$$
\left[\partial \nu_{k}^{R} / \partial n\right]=-\left[\Phi_{0}\right] \tilde{\Omega}_{k}^{R}
$$

Condition (74) can also be simplified by writing $\nabla_{s} \tilde{\Omega}_{k}^{R}=\left(\nabla_{s} \tilde{\Omega}_{k}^{R} \cdot \boldsymbol{t}\right) \boldsymbol{t}+\left(\nabla_{s} \tilde{\Omega}_{k}^{R} \cdot \boldsymbol{l}\right) \boldsymbol{l}$ and taking the cross product with $\boldsymbol{l}$ to obtain

$$
\sum_{\mathcal{I} \in\{A B, B S, S A\}} \sigma_{\mathcal{I}}\left(\nabla_{s} \tilde{\Omega}_{k}^{R} \cdot \boldsymbol{t}_{\mathcal{I}}\right) \boldsymbol{n}_{\mathcal{I}}=0, \quad \boldsymbol{x} \in \partial \partial \Omega .
$$

The underlying translational symmetry implies that the linearized problem (71)(74) also has solutions $\nu_{k}^{T}=\partial \nu / \partial x_{k}, k=1,2,3$, where the corresponding interface displacements are $\tilde{\Omega}_{k}^{T}=\boldsymbol{n}_{k}$. Regarding (71)-(74) as a homogeneous linear system, $\tilde{\Omega}_{k}^{R}$ and $\tilde{\Omega}_{k}^{T}$ are simply the eigenfunctions in the kernel of the linearized operator and will be utilized in a solvability argument. These calculations require the far field behavior of the field $\nu$ associated with the eigenfunctions, which using (68) and (70) give

$$
\nu_{k}^{R} \sim \frac{|\boldsymbol{p}| e^{k} \cdot \boldsymbol{x}}{|\boldsymbol{x}|^{3}}, \quad \boldsymbol{x} \rightarrow \infty,
$$

and

$$
\nu_{k}^{T} \sim \frac{\boldsymbol{p}_{k}|\boldsymbol{x}|^{2}-3(\boldsymbol{p} \cdot \boldsymbol{x}) \boldsymbol{x}_{k}}{|\boldsymbol{x}|^{5}}, \quad \boldsymbol{x} \rightarrow \infty .
$$

4.2. Rotation dynamics. We will now show that the collective effect of dipole fields generated by equilibrium configurations induces particle rotation. This is done by extending the analysis of section 3.1, incorporating higher expansion terms and longer timescales. It is supposed that a collection of particle domains $\left\{\Omega^{i}\right\}$, identical up to rotation, has reached diffusive equilibrium, in the sense that they have a common value of $\mu_{\infty}$. Beyond this point, $\mu$ remains constant and is suppressed in what follows. 
After expanding $\nu$ in powers of $\varepsilon$, it is found that $\nu_{0}$ is just the equilibrium value, and

$$
\nu_{0} \sim \frac{\boldsymbol{p}^{i} \cdot \boldsymbol{x}}{|\boldsymbol{x}|^{3}}, \quad \boldsymbol{x} \rightarrow \infty
$$

The corresponding orientations are $\boldsymbol{d}^{i}(T)$, where $T=\varepsilon^{3} t$ is a slow timescale. The interface displacements (relative to the equilibrium configuration) are also expanded as $\tilde{\Omega}=\epsilon \tilde{\Omega}_{1}+\epsilon^{2} \tilde{\Omega}_{2}+\epsilon^{3} \tilde{\Omega}_{3}+\epsilon^{4} \tilde{\Omega}_{4}+\cdots$.

The expansion using the outer coordinate is $\nu(\boldsymbol{y})=\varepsilon^{2} \nu_{2}(\boldsymbol{y})+\varepsilon^{3} \nu_{3}(\boldsymbol{y})+\cdots$, where the first nontrivial order solves $\Delta \nu_{2}(\boldsymbol{y})=0$. By matching,

$$
\nu_{2}(\boldsymbol{y}) \sim \frac{\boldsymbol{p}^{i} \cdot\left(\boldsymbol{y}-\boldsymbol{y}^{i}\right)}{\left|\boldsymbol{y}-\boldsymbol{y}^{i}\right|^{3}}, \quad \boldsymbol{y} \rightarrow \boldsymbol{y}^{i} .
$$

The solution using the Green's function defined by (46) is

$$
\nu_{2}(\boldsymbol{y})=-4 \pi \sum_{i=1}^{N} \nabla G\left(\boldsymbol{y}-\boldsymbol{y}^{i}\right) \cdot \boldsymbol{p}^{i}
$$

Expanding for $\boldsymbol{y} \approx \boldsymbol{y}^{i}$ leads to

$$
\begin{aligned}
\nu_{2}(\boldsymbol{y}) \sim & \frac{\boldsymbol{p}^{i} \cdot\left(\boldsymbol{y}-\boldsymbol{y}^{i}\right)}{\left|\boldsymbol{y}-\boldsymbol{y}^{i}\right|^{3}}-4 \pi \sum_{j=1, j \neq i}^{N} \nabla G\left(\boldsymbol{y}^{i}-\boldsymbol{y}^{j}\right) \cdot \boldsymbol{p}^{j} \\
& -4 \pi \sum_{j=1, j \neq i}^{N} \nabla^{2} G\left(\boldsymbol{y}^{i}-\boldsymbol{y}^{j}\right) \cdot \boldsymbol{p}^{j} \cdot\left(\boldsymbol{y}-\boldsymbol{y}^{i}\right) \\
& -2 \pi \sum_{j=1, j \neq i}^{N} \nabla^{3} G\left(\boldsymbol{y}^{i}-\boldsymbol{y}^{j}\right) \cdot \boldsymbol{p}^{j} \cdot\left(\boldsymbol{y}-\boldsymbol{y}^{i}\right) \cdot\left(\boldsymbol{y}-\boldsymbol{y}^{i}\right), \quad \boldsymbol{y} \rightarrow \boldsymbol{y}^{i} .
\end{aligned}
$$

Matching back to the inner expansion means that

$$
\nu_{3}(\boldsymbol{x}) \sim-4 \pi \sum_{j=1, j \neq i}^{N} \nabla^{2} G\left(\boldsymbol{y}^{i}-\boldsymbol{y}^{j}\right) \cdot \boldsymbol{p}^{j} \cdot \boldsymbol{x} \equiv \boldsymbol{\pi}^{i} \cdot \boldsymbol{x}, \quad \boldsymbol{x} \rightarrow \infty .
$$

For the inner expansion, it is found that $\nu_{j}(\boldsymbol{x})=0$ for $j=1,2$, and the problem at $O\left(\varepsilon^{3}\right)$ is

$$
\begin{aligned}
\Delta \nu_{3} & =0, \quad \boldsymbol{x} \in \Omega_{S} \cup \Omega_{A} \cup \Omega_{B}, \\
\nu_{3}\left[\Phi_{0}\right] & =\left\{\left(-\partial \nu_{0} / \partial n\right)+\sigma_{\mathcal{I}}\left(\Delta_{s}+\kappa_{1}^{2}+\kappa_{2}^{2}\right)\right\} \Omega_{3}, \quad \boldsymbol{x} \in \partial \Omega_{\mathcal{I}}, \\
{\left[\Phi_{0}\right] \frac{d \Omega}{d t} } & =-\left[\partial \nu_{3} / \partial n+\left(\partial^{2} \nu_{0} / \partial n^{2}\right) \tilde{\Omega}_{k}^{R}\right], \quad \boldsymbol{x} \in \partial \Omega_{\mathcal{I}}, \\
\sum_{\mathcal{I} \in\{A B, B S, S A\}} \sigma_{\mathcal{I}} \nabla_{s} \Omega_{3}(\mathcal{I}) & =0, \quad \boldsymbol{x} \in \partial \partial \Omega,
\end{aligned}
$$

supplemented with the far field condition (85). The interface motion induced by rotation is

$$
\frac{d \Omega}{d t} \equiv\left(\frac{d \boldsymbol{d}^{i}}{d T} \cdot \boldsymbol{e}^{1}\right) \tilde{\Omega}_{1}^{R}+\left(\frac{d \boldsymbol{d}^{i}}{d T} \cdot \boldsymbol{e}^{2}\right) \tilde{\Omega}_{2}^{R} .
$$

Copyright (c) by SIAM. Unauthorized reproduction of this article is prohibited. 
Solvability of the linear system (86)-(89) proceeds by multiplying (86) by $\nu_{k}^{R}=\nu_{k}^{R}\left(\boldsymbol{x} ; \boldsymbol{d}^{i}\right)$, integrating over a ball $B(r)$ of radius $r$, and taking $r \rightarrow \infty$. This gives

$$
\int_{\partial \Omega} \nu_{k}^{R}\left[\partial \nu_{3} / \partial n\right]-\nu_{3}\left[\partial \nu_{k}^{R} / \partial n\right] d \boldsymbol{x}+\int_{\partial B(r)} \nu_{k}^{R}\left(\partial \nu_{3} / \partial n\right)-\nu_{3}\left(\partial \nu_{k}^{R} / \partial n\right) d \boldsymbol{x}=0
$$

by Green's identity. Evaluation of the latter integral as $r \rightarrow \infty$ uses (79) and (85), giving

$$
\int_{\partial B(r)} \nu_{k}^{R}\left(\partial \nu_{3} / \partial n\right)-\nu_{3}\left(\partial \nu_{k}^{R} / \partial n\right) d \boldsymbol{x}=4 \pi\left|p^{i}\right|\left(\boldsymbol{\pi}^{i} \cdot \boldsymbol{e}^{k}\right) .
$$

The first integral in (91) can be written as

$$
\begin{aligned}
\int_{\partial \Omega} \nu_{k}^{R}\left[\partial \nu_{3} / \partial n\right]-\nu_{3}\left[\partial \nu_{k}^{R} / \partial n\right] d \boldsymbol{x} & =-\int_{\partial \Omega}\left[\Phi_{0}\right] \frac{d \Omega}{d t} \nu_{k}^{R} d \boldsymbol{x}-I, \\
I & \equiv \int_{\partial \Omega}\left[\Phi_{0}\right]\left(\nu_{k}^{R} \Omega_{3}-\nu_{3} \tilde{\Omega}_{k}^{R}\right) d \boldsymbol{x} .
\end{aligned}
$$

The integral term $I$ can be shown to vanish. Using (77) and (88), this term can be written as

$$
I=\int_{\partial \Omega} \sigma\left(\tilde{\Omega}_{k}^{R} \Delta_{s} \Omega_{3}-\Omega_{3} \Delta_{s} \tilde{\Omega}_{k}^{R}\right) d \boldsymbol{x}
$$

with $\sigma=\sigma_{\mathcal{I}}$ on each corresponding interface in $\partial \Omega$. Application of Green's identity to manifolds in $\partial \Omega$ means that (95) reduces to integrals along three-phase lines:

$$
I=\int_{\partial \partial \Omega} \sum_{\mathcal{I} \in\{A B, B S, S A\}} \sigma_{\mathcal{I}}\left[\left(\nabla_{s} \Omega_{3}(\mathcal{I}) \cdot \boldsymbol{t}_{\mathcal{I}}\right) \tilde{\Omega}_{k}^{R}(\mathcal{I})-\left(\nabla_{s} \tilde{\Omega}_{k}^{R}(\mathcal{I}) \cdot \boldsymbol{t}_{\mathcal{I}}\right) \Omega_{3}(\mathcal{I})\right] d \boldsymbol{x}
$$

Conditions (26), (78), and (89) imply

$$
\sum_{\mathcal{I} \in\{A B, B S, S A\}} \sigma_{\mathcal{I}}\left(\nabla_{s} \Omega_{3}(\mathcal{I}) \cdot \boldsymbol{t}_{\mathcal{I}}\right) \tilde{\Omega}_{k}^{R}(\mathcal{I})=\sum_{\mathcal{I} \in\{A B, B S, S A\}} \sigma_{\mathcal{I}}\left(\nabla_{s} \Omega_{3}(\mathcal{I}) \cdot \boldsymbol{t}_{\mathcal{I}}\right) \boldsymbol{q} \cdot \boldsymbol{n}_{\mathcal{I}}=0
$$

A similar expression holds for the second term in (96), which means that $I$ is zero as claimed.

The other integral on the right-hand side in (93) can be written using (77) as

$$
\begin{aligned}
-\int_{\partial \Omega}\left[\Phi_{0}\right] \frac{\boldsymbol{d} \Omega}{d T} \nu_{k}^{R} d \boldsymbol{x} & =\sum_{j=1}^{2}\left(\frac{d \boldsymbol{d}^{i}}{d T} \cdot \boldsymbol{e}^{j}\right) \int_{\partial \Omega}\left[\partial \nu_{j}^{R} / \partial n\right] \nu_{k}^{R} d \boldsymbol{x} \\
& =-\sum_{j=1}^{2}\left(\frac{d \boldsymbol{d}^{i}}{d T} \cdot \boldsymbol{e}^{j}\right) \int_{\mathbb{R}^{3}} \nabla \nu_{j}^{R} \cdot \nabla \nu_{k}^{R} d \boldsymbol{x} .
\end{aligned}
$$

4.3. Alignment effects. Using expressions (92) and (98) in (91), the rotational dynamics may be written as

$$
\boldsymbol{M}^{i} \boldsymbol{E} \frac{d \boldsymbol{d}^{i}}{d T}=4 \pi\left|\boldsymbol{p}^{i}\right| \boldsymbol{E} \boldsymbol{\pi}^{i}
$$

Copyright $@$ by SIAM. Unauthorized reproduction of this article is prohibited. 
where $\boldsymbol{E}_{k j}=\boldsymbol{e}_{j}^{k}$ and

$$
\boldsymbol{M}_{k j}^{i}=\int_{\mathbb{R}^{3}} \nabla \nu_{j}^{R} \cdot \nabla \nu_{k}^{R} d \boldsymbol{x} .
$$

Equation (100) can be written in a form independent of the choice of basis vectors $e^{1,2}$ as

$$
\frac{d \boldsymbol{d}^{i}}{d T}=4 \pi\left|\boldsymbol{p}^{i}\right| \tilde{\boldsymbol{M}}^{i} \boldsymbol{\pi}^{i}
$$

where the matrix $\tilde{\boldsymbol{M}}^{i}=\boldsymbol{E}^{T}\left(\boldsymbol{M}^{i}\right)^{-1} \boldsymbol{E}$. Note that both $\boldsymbol{M}^{i}$ and $\tilde{\boldsymbol{M}}^{i}$ are nonnegative definite.

Equation (102) represents an interacting system of particles which rotate in response to their neighbor's orientation. This system has a natural dissipated energy

$$
E_{R}=\frac{1}{2} \sum_{i=1}^{N} \sum_{j=1, j \neq i}^{N} \nabla^{2} G\left(\boldsymbol{y}^{i}-\boldsymbol{y}^{j}\right) \cdot \boldsymbol{p}^{i} \cdot \boldsymbol{p}^{j}
$$

Using (102), one has

$$
\frac{d E_{R}}{d T}=-\frac{1}{4 \pi} \sum_{i=1}^{N}\left|\boldsymbol{p}^{i}\right| \boldsymbol{\pi}^{i} \cdot \frac{d \boldsymbol{d}^{i}}{d T}=-\sum_{i=1}^{N}\left|\boldsymbol{p}^{i}\right|^{2} \tilde{\boldsymbol{M}}^{i} \cdot \boldsymbol{\pi}^{i} \cdot \boldsymbol{\pi}^{i} \leq 0 .
$$

For large systems where boundary effects are negligible, the Green's function is well approximated by the free-space Green's function $G \approx-1 /(4 \pi|\boldsymbol{x}|)$ which allows (103) to be written as

$$
E_{R} \approx \frac{1}{8 \pi} \sum_{i=1}^{N} \sum_{j=1, j \neq i}^{N} \frac{\boldsymbol{p}^{i} \cdot \boldsymbol{p}^{j}-3\left(\boldsymbol{p}^{i} \cdot \boldsymbol{\Delta}^{i j}\right)\left(\boldsymbol{p}^{j} \cdot \boldsymbol{\Delta}^{i j}\right)}{\left|\boldsymbol{y}^{i}-\boldsymbol{y}^{j}\right|^{3}}, \quad \boldsymbol{\Delta}^{i j} \equiv \frac{\boldsymbol{y}^{i}-\boldsymbol{y}^{j}}{\left|\boldsymbol{y}^{i}-\boldsymbol{y}^{j}\right|} .
$$

Since the interaction has a strongly decaying character, it is illuminating to consider a system with only two particles. In this case, energy is proportional to $\boldsymbol{p}^{1} \cdot \boldsymbol{p}^{2}-3\left(\boldsymbol{p}^{1} \cdot \boldsymbol{\Delta}^{12}\right)\left(\boldsymbol{p}^{2} \cdot \boldsymbol{\Delta}^{12}\right)$. Subject to fixed values of $\left|\boldsymbol{p}^{i}\right|$ and $\left|\boldsymbol{p}^{j}\right|$, it is straightforward to check that there are two global minima given by $\boldsymbol{p}^{1}=\boldsymbol{p}^{2}= \pm \boldsymbol{\Delta}^{12}$. In other words, the dipole moments preferentially align with the displacement vector between particles. This effect is tested numerically in section 5.2.

4.4. Translation dynamics. Using (84), matching to the inner expansion provides the far field behavior

$$
\nu_{4}(x) \sim-2 \pi \sum_{j=1, j \neq i}^{N} \nabla^{3} G\left(\boldsymbol{y}^{i}-\boldsymbol{y}^{j}\right) \cdot \boldsymbol{p}^{j} \cdot \boldsymbol{x} \cdot \boldsymbol{x} \equiv \boldsymbol{\Pi}^{i} \cdot \boldsymbol{x} \cdot \boldsymbol{x}, \quad \boldsymbol{x} \rightarrow \boldsymbol{x}^{i} .
$$

On the very slow timescale $T_{2}=\varepsilon^{5} t$, the rotational dynamics has equilibrated, but centers of mass $\boldsymbol{y}^{i}=\boldsymbol{y}^{i}\left(T_{2}\right)$ may be influenced by the far field behavior of $\nu_{4}$. This order in the expansion solves a system similar to (86)-(89), with (88) replaced by

$$
\left[\Phi_{0}\right] \frac{d \Omega}{d T_{2}}=-\left[\partial \nu_{4} / \partial n+\left(\partial^{2} \nu_{0} / \partial n^{2}\right) \Omega_{4}\right], \quad \frac{d \Omega}{d T_{2}} \equiv-\sum_{k=1}^{3} \nu_{k}^{T} \cdot \frac{d \boldsymbol{y}_{k}^{i}}{d T_{2}}
$$


The solvability argument is essentially identical to that above. After multiplying the Laplace equation by eigenfunctions $\nu_{k}^{T}$, one arrives at

$$
\int_{\partial \Omega} \nu_{k}^{T}\left[\partial \nu_{4} / \partial n\right]-\nu_{4}\left[\partial \nu_{k}^{T} / \partial n\right] d \boldsymbol{x}+\int_{\partial B(r)} \nu_{k}^{T}\left(\partial \nu_{4} / \partial n\right)-\nu_{4}\left(\partial \nu_{k}^{T} / \partial n\right) d \boldsymbol{x}=0
$$

In this case, the latter integral can be computed using (80) and (106), giving

$$
\int_{\partial B(r)} \nu_{k}^{T}\left(\partial \nu_{4} / \partial n\right)-\nu_{4}\left(\partial \nu_{k}^{T} / \partial n\right) d \boldsymbol{x} \sim-2 \pi\left(\boldsymbol{\Pi}^{i} \boldsymbol{p}^{i}\right)_{k}, \quad r \rightarrow \infty .
$$

The first integral in (108) uses a computation analogous to (93) and (98), leading to

$$
\int_{\partial \Omega} \nu_{k}^{T}\left[\partial \nu_{4} / \partial n\right]-\nu_{4}\left[\partial \nu_{k}^{T} / \partial n\right] d \boldsymbol{x}=\sum_{l=1}^{3} \frac{d \boldsymbol{y}_{l}^{i}}{d T_{2}} \int_{\mathbb{R}^{3}} \nabla \nu_{k}^{T} \cdot \nabla \nu_{l}^{T} d \boldsymbol{x} .
$$

Combining the previous two expressions, the translation dynamics can be written compactly as

$$
\frac{d \boldsymbol{y}^{i}}{d T_{2}}=2 \pi\left(\boldsymbol{M}^{i}\right)^{-1} \boldsymbol{\Pi}^{i} \boldsymbol{p}^{i}, \quad \boldsymbol{M}_{k l}=\int_{\mathbb{R}^{3}} \nabla \nu_{k}^{T} \cdot \nabla \nu_{l}^{T} d \boldsymbol{x} .
$$

The energy defined in (103) is also dissipated by the dynamics (111), since

$$
\frac{d E_{R}}{d T_{2}}=-\frac{1}{2 \pi} \sum_{i=1}^{N} \boldsymbol{\Pi}^{i} \boldsymbol{p}^{i} \frac{d \boldsymbol{y}^{i}}{d T_{2}}=-\sum_{i=1}^{N}\left(\boldsymbol{M}^{-1} \boldsymbol{\Pi}^{i} \boldsymbol{p}^{i}\right) \cdot\left(\boldsymbol{\Pi}^{i} \boldsymbol{p}^{i}\right) \leq 0 .
$$

It is again instructive to consider the interaction among only two particles. Since orientational alignment is achieved on a faster timescale, the terms in (105) are proportional to $-\left|\boldsymbol{y}^{1}-\boldsymbol{y}^{2}\right|^{3}$. This means that there is an energetic preference for aligned particles to attract, which will be illustrated in section 5.2.

5. Numerical simulation. This section illustrates and confirms many of the preceding analytical results using numerical computation of the diffuse interface model (5). The numerical methods employed use a spectral spatial discretization and guarantee that the discrete version of the energy (2) is dissipated. The other details are fully described elsewhere [57]. For simplicity, the surface energy parameters were all chosen to be unity, with the exception of the computations of section 5.2.

5.1. Competitive dynamics. Simulations with a computational grid of size $256^{3}$ were conducted to observe the formation and interaction of nanoparticle structures. In order to generate spatially distinct domains, initial conditions were chosen to represent small randomly placed spherical patches. To do this, $\Psi$ was set to $1 / 2$ within these patches and was set to zero outside. The variable $\Phi$ was chosen to be small and random to initiate phase separation. When patches were chosen to be too small, they failed to nucleate domains of heterogeneous polymer composition and subsequently vanished. Trial and error was used to set the patch size to be just large enough so this does not occur.

Figure 4 shows the evolution in the case where the A-monomer volume fraction was $f=0.5$. Initially, mostly two-bubble domains are formed and compete via Ostwald ripening as predicted. Structures with more than two subdomains were continuously created either by morphological transitions or coalescence events and 

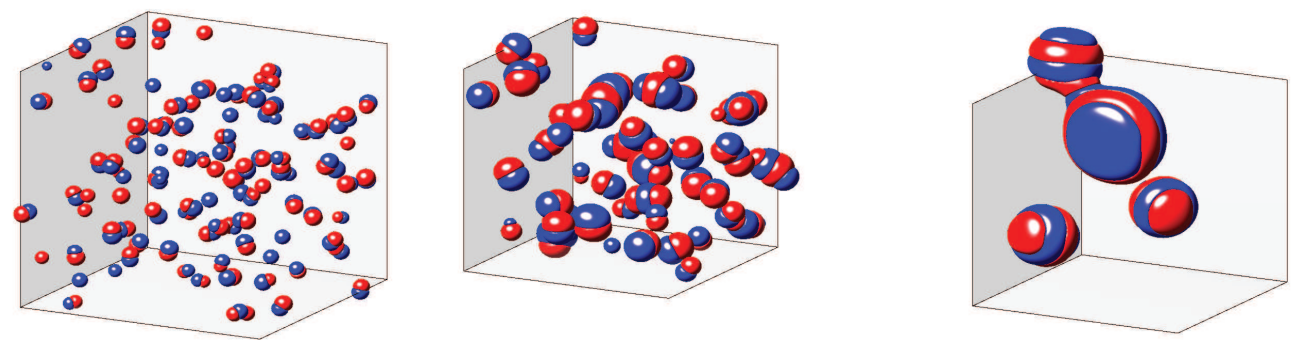

FIG. 4. Three-dimensional simulations at times $t=280,3.5 \times 10^{3}, 7.3 \times 10^{4}$. The surface energy parameters are all set to one, and the volume fraction parameter was $f=0.5$. The final state (not shown) is a single multilayered nanoparticle.
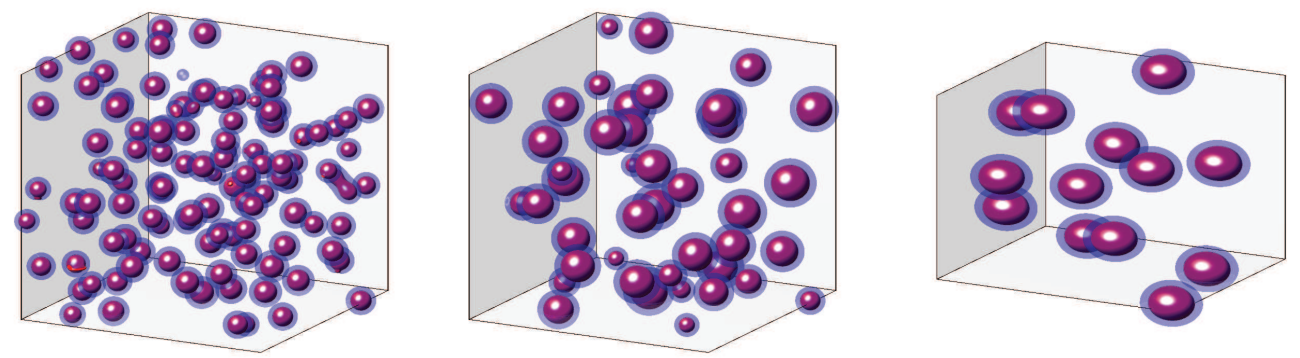

FIG. 5. Three-dimensional simulations at times $t=700,4.2 \times 10^{3}, 2.0 \times 10^{4}$. The parameters are the same as in Figure 4, except $f=0.35$. After a ripening phase, all micelles equilibrate to the same radius (far right).

ultimately outcompete two-bubble domains. This process continues until just a single multilayered nanoparticle remains.

Figure 5 shows the evolution with the same parameters, except the monomer volume fraction was $f=0.35$ instead. In this case, particles take the form of twolayered micelles initially. Ripening of domain structures is observed for a while, but this eventually halts once a critical size is reached, leaving many equally sized micelles.

5.2. Rotation and translation. The theoretical predictions of section 4 indicate that late stage dynamics of coexisting particles without radial symmetry is characterized by rotational alignment and subsequent pairwise attraction. To demonstrate this, two-bubble particles are natural candidates. With equal surface energies, however, we have seen that these will compete by Ostwald ripening and no late stage coexistence is possible. We have found, on the other hand, that the choice of surface energy parameters $\sigma_{A B}=2$ and $\sigma_{A S}=\sigma_{B S}=1$ (with $f=0.5$ ) does yield a stable collection of two-bubble particles.

A simple numerical illustration confirms the qualitative nature of the theoretical findings. Initial conditions were chosen to be two spherical domains, each subdivided along a diameter into $A$ - and $B$-phase subdomains where the orientation of each particle is different. These regions quickly develop into two-bubble domain structures, each of the same size, and then exhibit rigid body motion.

The results are shown in Figure 6. After the two structures have obtained their equilibrium shape, they each rotate, largely without any other movement, until both 

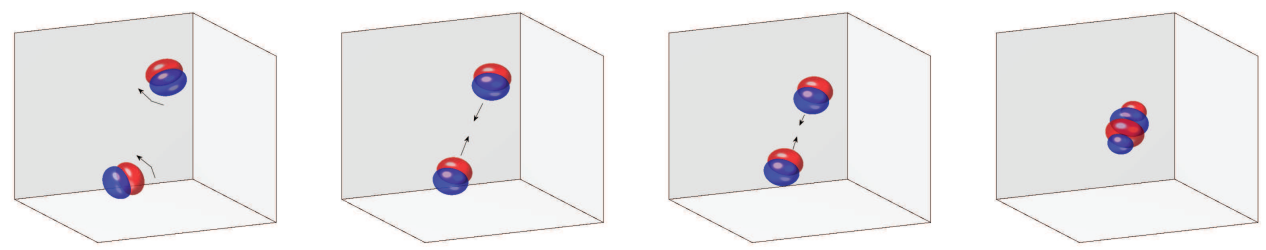

FIG. 6. Simulation of late stage interaction dynamics, where the arrows indicate the instantaneous dynamics. Initially, particles rotate (left) until they align (left middle) and migrate toward each other (right middle) until they merge (right).

axes of symmetry are aligned with a line drawn between particles. The particles then drift toward one another, and finally merge, creating a four-domain bubble.

5.3. Behavior in two dimensions and dynamic scaling. To acquire meaningful statistics of dynamical scaling phenomena, computations with a very large number of particles are required. Unfortunately, this regime is (at least presently) numerically inaccessible in three dimensions. On the other hand, simulations in two dimensions reveal phenomena similar to those in three and allow for much larger system sizes to be investigated.

Although simple morphologies analogous to micelles and multiple bubbles are seen in two dimensions as well, it is also feasible to compute a much wider variety of complex equilibria. To illustrate this, steady states were found using the gradient descent dynamics (5), with initial conditions where $\Psi=1 / 2$ on a circular patch of adjustable radius and where $\Phi$ was chosen to be small and random. In addition, surface energies were all set to unity and the volume fraction parameter was $f=0.5$. The relationship $\mu_{\infty}(M)$ was computed for numerous particles of different sizes as in section 2.4, setting $\mu_{\infty}=\partial W / \partial \Psi$ far away from interfaces and finding $M$ from numerical integration of $\Psi$ within the polymer domain region. Figure 7 shows the computed relationship, along with a nonlinear least squares fit of the data and plots of representative equilibria. Consistent with the conjecture in section 2.2, subdomains appear to have a distinct characteristic width.

The dynamics of a large number of particles was also investigated, using a computational grid of size $4048^{2}$. The behavior with polymer volume fraction $f=0.5$ and all surface energies set to one is shown in Figure 8. As in three dimensions, phase segregation initially produces two-bubble domains. As ripening ensues, multiple bubble structures appear. The final result (not shown) is a single large particle with a lamellar substructure. Asymmetric mixtures with volume fraction $f=0.35$ and equal surface energies were also investigated (Figure 9). As expected, ripening behavior eventually gives way to equilibration of micelle radii.

To quantify dynamic scaling, statistics on domain size were computed. Polymerrich domains were identified by finding regions where the order parameter $\Psi>0.1$, and their effective radius was then found by computing the square root of the corresponding area. The average radius was computed at each simulation time to determine scaling or nonscaling of domain sizes.

Figure 10 shows the average radius $R(t)$ as a function of time for the simulations in Figures 8 and 9 . The case of equal surface energies (left panel) demonstrates fairly consistent power-law scaling for times $<10^{5}$, at which point the number of particles is less than 10 and they have acquired complicated morphologies. Within this time 


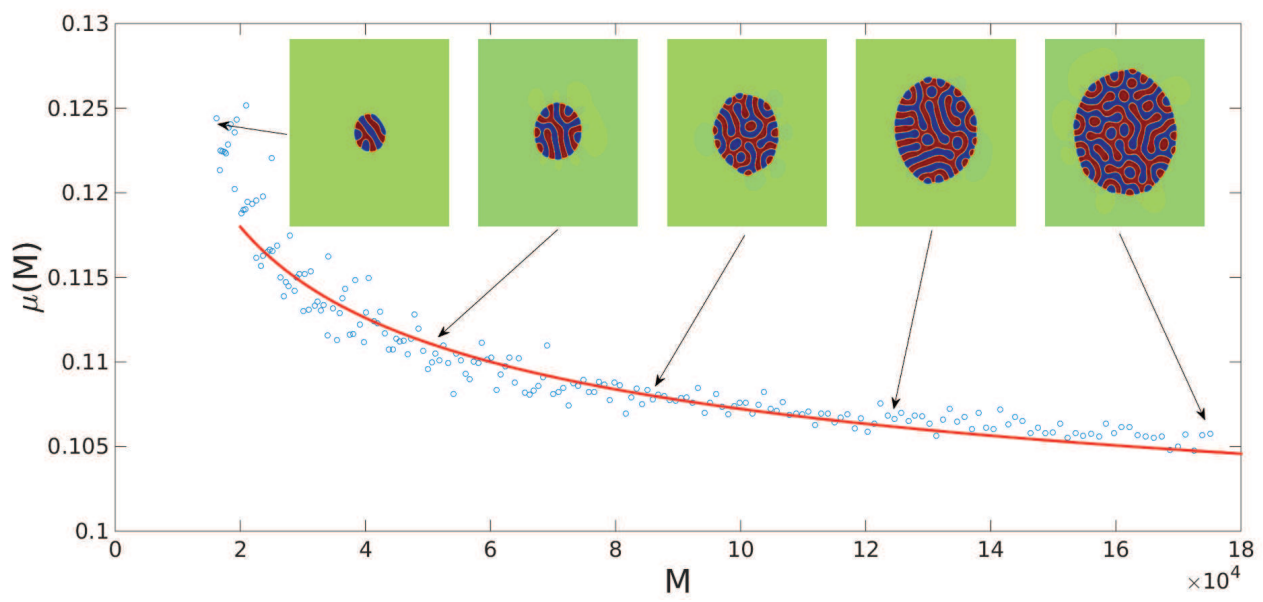

FIG. 7. Relationship between equilibrium chemical potential $\mu_{\infty}$ and size parameter $M$ for numerous two-dimensional equilibria. A nonlinear least squares fit of the form $\mu=A+B r^{\gamma}$ is shown, where $A=0.093, B=0.85, \gamma=-0.35$. Domain patterns for several of the computed equilibria are also shown.
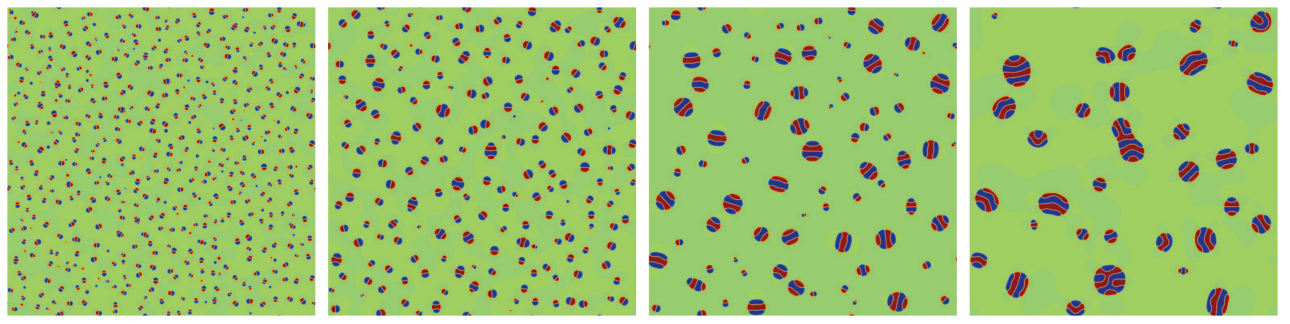

FIG. 8. Simulation for equal surface energies and volume parameter $f=0.5$ at times $t=780,6.9 \times 10^{3}, 2.6 \times 10^{4}, 8.9 \times 10^{4}$. Particles undergo Ostwald ripening as well as encounter morphological transitions as a result of instability and coalescence.
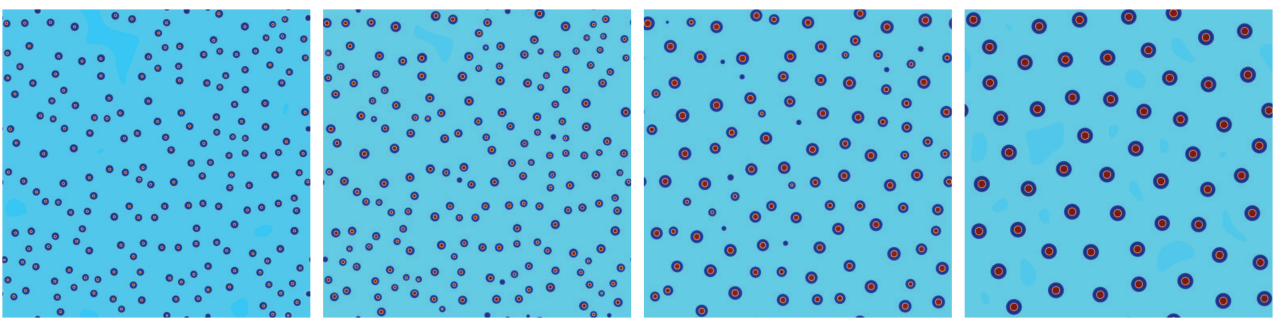

FIG. 9. Simulation at times $t=440,2.0 \times 10^{3}, 1.3 \times 10^{4}, 1.20 \times 10^{6}$, where the parameters are like Figure 8, except the polymer volume fraction was $f=0.35$.

frame, a power law fit $R \sim t^{\alpha}$ was found with $\alpha=0.33$. Statistics for the other scenario for which ripening halts are shown in Figure 10(right). In this case, there is a transient regime where the scaling of a radius is consistent with the $t^{1 / 5}$-power law.

6. Discussion and conclusions. This paper has explored a broad class of dynamical phenomena in multiphase density functional models. This includes formation 

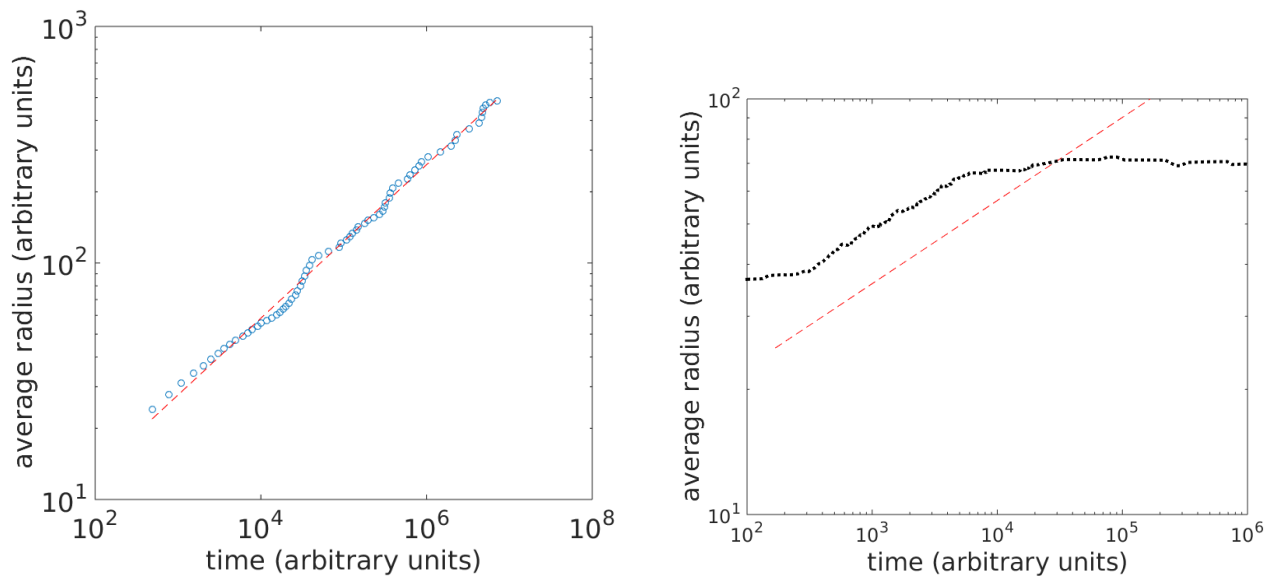

FIG. 10. Left: average particle radius as a function of time for the simulation in Figure 8. The dashed line corresponds to a linear regression fit with slope $\approx 0.33$. Right: average radius as a function of time for the case of unequal composition as in Figure 9 (dotted). The dashed line is for reference and has slope $1 / 5$.

of topologically diverse equilibria, multiparticle stability and instability, and interaction leading to rotation and translation. Disparate length- and timescales were exploited to explicitly calculate the evolution of a reduced set of configurational degrees of freedom. These simplified models demonstrate qualitative aspects of nanoparticle self-assembly, such as the preference of certain morphologies and sizes. They also show how collective effects may influence the overall trajectory of pattern development.

Some surprising features of the dynamics were found, including the possibility of stable multiparticle assemblies and orientational alignment. These effects are important to the processing of block copolymer materials, where control over final microstructure is desirable $[18,58]$.

Our computational results exhibiting a wide class of possible equilibrium morphologies run parallel to Avalos et al. [9]. They utilize a similar system of coupled Cahn-Hilliard-type equations but with phenomenological parameters in the bulk potential. Both their framework and ours appear capable of reproducing experimentally observed structures.

The asymptotic analysis used herein extends the classical LSW approximation for binary phase mixtures. Previously, two-phase systems with nonlocal repulsion were also studied with this approach $[56,59,60]$. The main finding of these investigations is that nonlocal energy terms stabilize ripening behavior and lead to patterned domain assemblies. In contrast, there is no long-range order at late stages here, even when ripening effects halt.

Competing morphologies in an amphiphilic system were studied by Dai and Promislow [61] (this is different from our system, where $A$ and $B$ phases are hydrophobic). In their case, spherical and toroidal morphologies are formed. Like our system, they compete diffusively by differences in chemical potential, leading to either extinction of spherical structures or coexistence of both types.

Recently, Wang, Ren, and Zhao [62] studied a system similar to ours but with nonlocal interaction among all three phases. They observe assemblies of two-bubble domain patterns which eventually establish both spatial and nematic (orientationally aligned) order. In contrast to our model, however, the nonlocal repulsion with the 
third phase modifies the domain interaction and prevents attraction and coalescence. This results in a final state comprised of a lattice of aligned domain patterns.

While our computations are limited to the choice of equal surface energies, it is expected that these parameters will also heavily influence preferred morphology. A complete taxonomy of morphological classes and the associated phase diagram would be valuable. This is a computationally challenging undertaking, since there are numerous physical parameters that influence equilibrium behavior. Combined with the theoretical results established here, this would be an important step toward the broad goal of engineering spontaneously assembled block copolymer structures.

\section{REFERENCES}

[1] F. S. Bates And G. H. Fredrickson, Block copolymers - designer soft materials, Phys. Today, 52 (1999), pp. 32-38.

[2] M. W. MAtsen AND F. S. BATES, Unifying weak- and strong-segregation block copolymer theories, Macromolecules, 29 (1996), pp. 1091-1098.

[3] I. W. Hamley, The Physics of Block Copolymers, Oxford Science Publications, 1998.

[4] A. Blanazs, S. P. ARmes, And A. J. RYAn, Self-assembled block copolymer aggregates: From micelles to vesicles and their biological applications, Macromol. Rapid Commun., 30 (2009), pp. 267-277.

[5] Y. Mai And A. Eisenberg, Self-assembly of block copolymers, Chem. Soc. Rev., 41 (2012), pp. $5969-5985$.

[6] K. I. Winey, E. L. Thomas, And L. J. Fetters, Isothermal morphology diagrams for binary blends of diblock copolymer and homopolymer, Macromolecules, 25 (1992), pp. 2645-2650.

[7] T. Hashimoto, S. Koizumi, And H. Hasegawa, Ordered structure in blends of block copolymers. 2. Self-assembly for immiscible lamella-forming copolymers, Macromolecules, 27 (1994), pp. 1562-1570.

[8] Z. Jin AND H. FAN, Self-assembly of nanostructured block copolymer nanoparticles, Soft Matter, 10 (2014), pp. 9212-9219.

[9] E. Avalos, T. Higuchi, T. Teramoto, H. Yabu, and Y. Nishiura, Frustrated phases under three-dimensional confinement simulated by a set of coupled Cahn-Hilliard equations, Soft Matter, 12 (2016), pp. 5905-5914.

[10] K. Zhang, L. GaO, Y. Chen, and Z. Yang, Onionlike spherical polymer composites with controlled dispersion of gold nanoclusters, Chem. Mater., 20 (2007), pp. 23-25.

[11] A. Rahikkala, A. J. Soininen, J. Ruokolainen, R. Mezzenga, J. Raula, and E. I. KaupPINEN, Self-assembly of ps-b-p4vp block copolymers of varying architectures in aerosol nanospheres, Soft Matter, 9 (2013), pp. 1492-1499.

[12] R. Deng, S. Liu, J. Li, Y. LiaO, J. TAO, AND J. Zhu, Mesoporous block copolymer nanoparticles with tailored structures by hydrogen-bonding-assisted self-assembly, Adv. Mater., 24 (2012), pp. 1889-1893.

[13] T. Higuchi, A. Tajima, K. Motoyoshi, H. Yabu, and M. Shimomura, Frustrated phases of block copolymers in nanoparticles, Angew. Chem., 120 (2008), pp. 8164-8166.

[14] S.-J. JEOn, G.-R. YI, AND S.-M. YANG, Cooperative assembly of block copolymers with deformable interfaces: Toward nanostructured particles, Adv. Mater., 20 (2008), pp. 41034108.

[15] K. Zhang, X. Yu, L. GaO, Y. Chen, And Z. Yang, Mesostructured spheres of organic/inorganic hybrid from gelable block copolymers and arched nano-objects thereof, Langmuir, 24 (2008), pp. 6542-6548.

[16] Z. Lu, G. LiU, AND F. LiU, Block copolymer microspheres containing intricate nanometer-sized segregation patterns, Macromolecules, 34 (2001), pp. 8814-8817.

[17] C.-S. Lim, J. H. Heo, M. S. You, S. H. Im, ANd K. J. Chae, Synthesis of $p s-b-p 2 v p$ di-block copolymer particles with internal structure via simple reprecipitation method, Macromol. Res., 22 (2014), pp. 324-328.

[18] M. Grzelczak, J. Vermant, E. M. Furst, and L. M. Liz-Marzán, Directed self-assembly of nanoparticles, ACS Nano, 4 (2010), pp. 3591-3605.

[19] E. Avalos, T. Teramoto, H. Komiyama, H. Yabu, and Y. Nishiura, Transformation of block copolymer nanoparticles from ellipsoids with striped lamellae into onionlike spheres and dynamical control via coupled Cahn-Hilliard equations, ACS Omega, 3 (2018), pp. 1304-1314.

Copyright $@$ by SIAM. Unauthorized reproduction of this article is prohibited. 
[20] I. Fortelnỳ, A. ZivnỲ, AND J. JuZA, Coarsening of the phase structure in immiscible polymer blends. Coalescence or Ostwald ripening?, J. Polym. Sci. B, 37 (1999), pp. 181-187.

[21] W.-S. Wang, L. Zhen, C.-Y. Xu, And W.-Z. ShaO, Aqueous solution synthesis of $\mathrm{Cd}(\mathrm{OH})_{2}$ hollow microspheres via Ostwald ripening and their conversion to $\mathrm{CdO}$ hollow microspheres, J. Phys. Chem. C, 112 (2008), pp. 14360-14366.

[22] S. KeßLER, K. Drese, AND F. SChmid, Simulating copolymeric nanoparticle assembly in the cosolvent method: How mixing rates control final particle sizes and morphologies, Polymer, 126 (2017), pp. 9-18.

[23] T. Онта AND A. Ito, Dynamics of phase separation in copolymer-homopolymer mixtures, Phys. Rev. E, 52 (1995), pp. 5250-5260.

[24] A. Iто, Domain patterns in copolymer-homopolymer mixtures, Phys. Rev. E, 58 (1998), pp. 6158-6165.

[25] T. Uneyama And M. Doi, Calculation of the micellar structure of polymer surfactant on the basis of the density functional theory, Macromolecules, 38 (2005), pp. 5817-5825.

[26] T. Uneyama, Density functional simulation of spontaneous formation of vesicle in block copolymer solutions, J. Chem. Phys., 126 (2007), 114902.

[27] K. Glasner and S. Orizaga, Multidimensional equilibria and their stability in copolymersolvent mixtures, Phys. D., 373 (2018), pp. 1-12.

[28] G. J. A. Sevink and A. V. Zvelindovsky, Self-assembly of complex vesicles, Macromolecules, 38 (2005), pp. $7502-7513$.

[29] X. He AND F. Schmid, Spontaneous formation of complex micelles from a homogeneous solution, Phys. Rev. Lett., 100 (2008), 137802.

[30] L. LeIBLER, Theory of microphase separation in block copolymers, Macromolecules, 13 (1980), pp. $1602-1617$.

[31] T. OHTA AND K. KaWASAKI, Equilibrium morphology of block copolymer melts, Macromolecules, 19 (1986), pp. 2621-2632.

[32] T. Ohta AND K. KaWASAKI, Comment on the free energy functional of block copolymer melts in the strong segregation limit, Macromolecules, 23 (1990), pp. 2413-2414.

[33] J. W. CAhn AND J. E. Hilliard, Free energy of a nonuniform system I. Interfacial free energy, J. Chem. Phys., 28 (1957), pp. 258-267.

[34] Y. Nishiura And I. Ohnishi, Some mathematical aspects of the micro-phase separation of diblock copolymers, Phys. D, 84 (1995), pp. 31-39.

[35] R. Choksi AND X. Ren, Diblock copolymer/homopolymer blends: Derivation of a density functional theory, Phys. D, 203 (2005), pp. 100-119.

[36] Y. van Gennip and M. A. Peletier, Copolymer-homopoymer blends: Global energy minimisation and global energy bounds, Calc. Var. Partial Differential Equations, 33 (2008), pp. $75-111$.

[37] Y. Van Gennip And M. A. Peletier, Stability of monolayers and bilayers in a copolymerhomopolymer blend model, Interfaces Free Bound., 11 (2009), pp. 331-373.

[38] K. GLasner, Multilayered equilibria in a density functional model of copolymer-solvent mixtures, SIAM J. Math. Anal., 49 (2017), pp. 1593-1620, https://doi.org/10.1137/ 16M1066129.

[39] X. Ren AND J. WeI, Concentrically layered energy equilibria of the di-block copolymer problem, European J. Appl. Math., 13 (2002), pp. 479-496.

[40] X. REN AND J. WeI, A double bubble in a ternary system with inhibitory long range interaction, Arch. Ration. Mech. Anal., 208 (2013), pp. 201-253.

[41] K. B. Glasner, Spatially localized structures in diblock copolymer mixtures, SIAM J. Appl. Math., 70 (2010), pp. 2045-2074, https://doi.org/10.1137/080743913.

[42] K. B. Glasner, Characterising the disordered state of block copolymers: Bifurcations of localised states and self-replication dynamics, European J. Appl. Math., 23 (2012), pp. 315341.

[43] X. Ren And C. Wang, A stationary core-shell assembly in a ternary inhibitory system, Discrete Cont. Dyn.-A, 37 (2017), pp. 983-1012.

[44] T. Ohta AND M. Nonomura, Elastic property of bilayer membrane in copolymer-homopolymer mixtures, Eur. Phys. J. B, 2 (1998), pp. 57-68.

[45] R. L. Pego, Front migration in the nonlinear Cahn-Hilliard equation, Proc. Roy. Soc. London Ser. A, 422 (1989), pp. 261-278.

[46] L. Bronsard, H. Garcke, and B. Stoth, A multi-phase Mullins-Sekerka system: Matched asymptotic expansions and an implicit time discretisation for the geometric evolution problem, Proc. Roy. Soc. Edinburgh Sect. A, 128 (1998), pp. 481-506.

[47] S. BALdo, Minimal interface criterion for phase transitions in mixtures of Cahn-Hilliard fluids, Ann. Inst. H. Poincaré Anal. Non Linéaire, 7 (1990), pp. 67-90.

Copyright (c) by SIAM. Unauthorized reproduction of this article is prohibited. 
[48] F. Boyer And C. Lapuerta, Study of a three component Cahn-Hilliard flow model, ESAIM Math. Model. Numer. Anal., 40 (2006), pp. 653-687.

[49] R. CHOKsI, Scaling laws in microphase separation of diblock copolymers, J. Nonlinear Sci., 11 (2001), pp. 223-236.

[50] X. REN AND J. WeI, On the multiplicity of solutions of two nonlocal variational problems, SIAM J. Math. Anal., 31 (2000), pp. 909-924, https://doi.org/10.1137/S0036141098348176.

[51] F. J. Almgren, Existence and regularity almost everywhere of solutions to elliptic variational problems with constraints, Mem. Amer. Math. Soc., 4 (1976), 165.

[52] M. Hutchings, F. Morgan, M. Ritoré, and A. Ros, Proof of the double bubble conjecture, Ann. of Math. (2), 155 (2002), pp. 459-489.

[53] I. M. Lifshitz AND V. V. Slyozov, The kinetics of precipitation from supersaturated solid solutions, J. Chem. Phys. Solids, 19 (1961), pp. 35-50.

[54] C. Wagner, Theorie for alterung von niederschlagen durch umlosen, Z. Elektrochemie, 65 (1961), pp. 581-594.

[55] P. Voorhees and L. Ratke, Growth and Coarsening, Springer, 2001.

[56] K. Glasner and R. Choksi, Coarsening and self-organization in dilute diblock copolymer melts and mixtures, Phys. D, 238 (2009), pp. 1241-1255.

[57] K. Glasner and S. Orizaga, Improving the accuracy of convexity splitting methods for gradient flow equations, J. Comput. Phys., 315 (2016), pp. 52-64.

[58] M. P. Stoykovich, M. Müller, S. O. Kim, H. H. Solak, E. W. Edwards, J. J. De Pablo, AND P. F. NEALEy, Directed assembly of block copolymer blends into nonregular deviceoriented structures, Science, 308 (2005), pp. 1442-1446.

[59] C. Sagui And R. C. Desai, Effects of long-range repulsive interactions on Ostwald ripening, Phys. Rev. E, 52 (1995), 2822-2840.

[60] X. Ren AND J. WeI, Spherical solutions to a nonlocal free boundary problem from diblock copolymer morphology, SIAM J. Math. Anal., 39 (2008), pp. 1497-1535, https://doi.org/ $10.1137 / 070690286$.

[61] S. Dai And K. Promislow, Competitive geometric evolution of amphiphilic interfaces, SIAM J. Math. Anal., 47 (2015), pp. 347-380, https://doi.org/10.1137/130941432.

[62] C. Wang, X. Ren, And Y. ZhaO, Bubble Assemblies in Ternary Systems with Long Range Interaction, preprint, https://arxiv.org/abs/1712.00724, 2017.

Copyright (C) by SIAM. Unauthorized reproduction of this article is prohibited. 